\title{
Extracellular and intracellular components of the impedance of neural tissue
}

Claude Bedard ${ }^{1}$, Charlotte Piette ${ }^{2}$, Laurent Venance ${ }^{2}$ and Alain Destexhe ${ }^{1}$

1: Paris-Saclay University, CNRS, Institute of Neuroscience (NeuroPSI), Gif sur Yvette, France

2: Center for Interdisciplinary Research in Biology (CIRB), Collège de France, CNRS, INSERM, PSL University, Paris, France

July 4, 2021

Condensed title: Neural tissue impedance

Keywords: Local field potential, Extracellular space, Ionic diffusion

The authors declare no conflict of interest. 


\begin{abstract}
Electric phenomena in brain tissue can be measured using extracellular potentials, such as the local field potential, or the electro-encephalogram. The interpretation of these signals depend on the electric structure and properties of extracellular media, but the measurements of these electric properties are subject to controversy. Some measurements point to a model where the extracellular medium is purely resistive, and thus parameters such as electric conductivity and permittivity should be independent of frequency. Other measurements point to a pronounced frequency dependence of these parameters, with scaling laws that are consistent with capacitive or diffusive effects. However, these experiments correspond to different preparations, and it is unclear how to correctly compare them. Here, we provide for the first time, impedance measurements in various preparations, for acute brain slices and primary cell cultures, and we compare to measurements using the same setup in artificial cerebrospinal fluid with no biological material. The measurements show that when the current flows across a cell membrane, the frequency dependence of the macroscopic impedance between intracellular and extracellular electrodes is significant, and cannot be captured by a model with resistive media. Fitting a mean-field model to the data shows that this frequency dependence could be explained by the ionic diffusion mainly associated to Debye layers surrounding the membranes. We conclude that neuronal membranes and their ionic environment induce strong deviations to resistivity, that should be taken into account to correctly interpret extracellular potentials generated by neurons.
\end{abstract}

\title{
Significance
}

The electro-encephalogram recorded at the scalp surface and local-field potentials recorded within neural tissue are generated by electric currents in neurons, and thus depend on the impedance of neural tissue. Different measured values were proposed, and it is currently unclear what is the real impedance of neural tissue. Here, we show that the impedance depends on the measurement technique. If the measurement is exclusively extracellular, the system appears as equivalent to a simple resistor. However, if the measurement includes an intracellular electrode, a more complex impedance is observed, because the current has to flow through the membrane, as happening in the brain. Thus, we provide an explanation for apparent disagreements, and indicate in which cases each impedance should be used. 


\section{Introduction}

The genesis of extracellular electric potentials in the brain depends on the electric properties of the extracellular medium. The exact nature of these electric properties is important, because non-resistive media will necessarily impose frequency-filtering properties to electric signals $[1,2]$ and therefore will influence any source localization. Early studies modeled the genesis of extracellular potentials assuming that the medium is analogous to a resistance [3]. Evidence for such resistive media was provided by different measurements $[4,5,6]$, while other measurements $[7,8,9]$ revealed a more complex situation, where the measured electric parameters displayed a dependence on frequency in contrast to the frequency-independence of resistive systems. Computational models showed that such a dependence of frequency can be obtained if there are strong spatial variations of conductivity and/or permittivity [10]. Further models showed that frequency-dependent electric parameters can also result from electric polarization of the medium [11], or from ionic diffusion [12].

The extracellular medium properties were also estimated indirectly by correlating intracellular and local field potentials [13] or by the frequency-dependence between electro-encephalogram and magneto-encephalogram signals [14]. Although a framework was proposed to explain the contradictory measurements [15], controversies still exist about the electric nature of the extracellular medium [15, 16].

The main problem accounting for controversies is that different experiments correspond to very different preparations, and it is not clear how to correctly compare them. For example, impedance was either measured intracellularly [9] or extracellularly [5, 6]. Could this account for discrepancies observed across studies ? In the present study, we provide for the first time, impedance measurements in different preparations, in acute brain slices, in primary cell cultures, and we compare to measurements using the same setup in artificial cerebrospinal fluid (ACSF).

\section{Materials and Methods}

\section{$2.1 \quad$ In vitro electrophysiology}

Animals. C57BL/6 and Swiss mice were housed by groups of 3-5 mice, in a $12 \mathrm{~h}$ light/dark cycle, with food and water available ad libitum. All experiments were performed in accordance with local animal welfare committee (Center for Interdisciplinary Research in Biology, CIRB, and Institut de Biologie Paris-Seine, IBPS, Ethical Committees) and EU guidelines (Directive 2010/63/EU). Every precaution was taken to minimize stress and the number of animals used in each series of experiments.

Neuronal primary cultures. The brains (from Swiss mice) were removed from day 14 embryos and were transferred to a plate containing PBS with $0.6 \%$ glucose. Striata were isolated under a stereomicroscope. Briefly, the hemispheres 
were cut with scissors and folded over, to visualize the dorsal striatum. Striata were mechanically dissociated by gently pipetting in PBS- $0.6 \%$ glucose. After decantation for $5 \mathrm{~min}$, cells were collected by centrifugation at $1000 \mathrm{x}$ g for 5 min. Cell pellets were resuspended in Neurobasal medium supplemented with B27 (Invitrogen, Thermo Fisher Scientific, Illkirch, France), 500 nM L-glutamine, 60 $\mu \mathrm{g} / \mathrm{ml}$ penicillin-streptomycin and $25 \mu \mathrm{M} \beta$-mercaptoethanol (Sigma, Saint-Quentin Fallavier, France) and then plated into 24 -well (1.8 x 105 cells per well) plates coated with $50 \mu \mathrm{g} / \mathrm{ml}$ poly-d-lysine (Sigma). After removal of the coating solution, cells were seeded in the Neurobasal medium on glass cover-slips and cultured at $37^{\circ} \mathrm{C}$ in a humidified atmosphere containing 95\% air and $5 \% \mathrm{CO}_{2}$. When placed in the recording chamber, the cell culture was initially superfused with a $95 \% \mathrm{O}_{2} / 5 \%$ $\mathrm{CO}_{2}$-bubbled Neurobasal medium, and then progressively diluted in ACSF solution. Patch-clamp recordings on neuronal cultures were made from day 3 to day 10 after seeding.

Brain slices preparation. Horizontal brain slices (from C57BL/6) with a thickness of $300 \mu \mathrm{m}$ were prepared from postnatal P30-40 mice using a vibrating blade microtome (VT1200S; Leica Biosystems, Nussloch, Germany). Brains were sliced in a $95 \% \mathrm{O}_{2} / 5 \% \mathrm{CO}_{2}$-bubbled, ice-cold cutting ACSF solution containing: $\mathrm{NaCl} 125 \mathrm{mM}, \mathrm{KCl} 2.5 \mathrm{mM}$, glucose $25 \mathrm{mM}, \mathrm{NaHCO}_{3} 25 \mathrm{mM}, \mathrm{NaH}_{2} \mathrm{PO}_{4} 1.25$ $\mathrm{mM}, \mathrm{CaCl}_{2} 1 \mathrm{mM}, \mathrm{MgCl}_{2} 1 \mathrm{mM}$, and pyruvic acid $1 \mathrm{mM}$ and then transferred into the same solution at $34^{\circ} \mathrm{C}$ for one hour before cell recording.

Whole-cell patch-clamp recordings and experimental set-up. Patchclamp recordings were performed in medium-sized spiny neurons and striatal interneurons of the dorsal striatum, combined with extracellular recording using a 2-3.5 M $\Omega$ patch-clamp glass pipette; The latter was located within a close vicinity $(\approx 5-10 \mu \mathrm{m})$ of the patched neuron (Fig. $1 \mathrm{~A})$. Borosilicate glass pipettes of $5-7 \mathrm{M} \Omega$ impedance contained for whole-cell recordings: K-gluconate $122 \mathrm{mM}, \mathrm{KCl} 13 \mathrm{mM}$, HEPES $10 \mathrm{mM}$, phosphocreatine $10 \mathrm{mM}$, ATP-Mg $4 \mathrm{mM}$, GTP-Na $0.3 \mathrm{mM}$, and EGTA $0.3 \mathrm{mM}$ (adjusted to $\mathrm{pH} 7.35$ with $\mathrm{KOH}$ ). The composition of the extracellular solution and inside the extracellular pipette was the same ACSF solution that was used for brain slices incubation. Signals were amplified using EPC10-2 amplifiers (HEKA Elektronik, Lambrecht, Germany) with a very high input impedance (1 T $\Omega$ ) to ensure there was no appreciable signal distortion imposed by the high impedance electrode $[17,18]$. All recordings were performed at $34^{\circ} \mathrm{C}$ using a temperature control system (Bath-Controller V; Luigs \& Neumann, Ratingen, Germany) and brain slices or primary cell cultures were continuously superfused at $2 \mathrm{ml} / \mathrm{min}$ with the extracellular solution. The extracellular solution used in the recording chamber had the very same ionic composition for all the experimental conditions. Neurons were visualized on a BX51WI microscope (Olympus, Rungis, France) using a 40x/0.80 water-immersion objective for localizing cells for whole-cell recordings and extracellular electrode positioning. Series resistance was not compensated. Current-clamp recordings were sampled at $50 \mathrm{kHz}$ using the Patchmaster v2x73 program (HEKA Elektronik).

White noise stimulation protocols. Frozen white noise stimuli were applied 

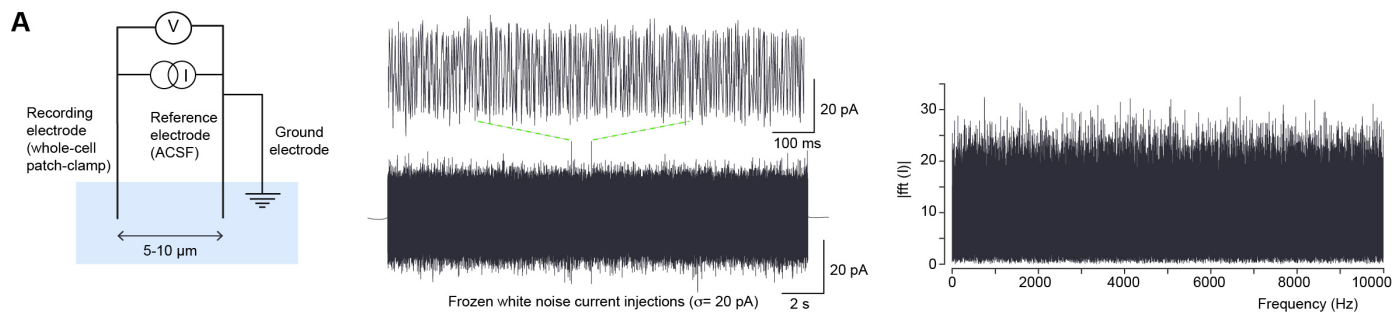

B

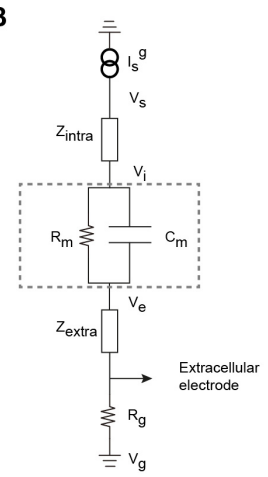

C

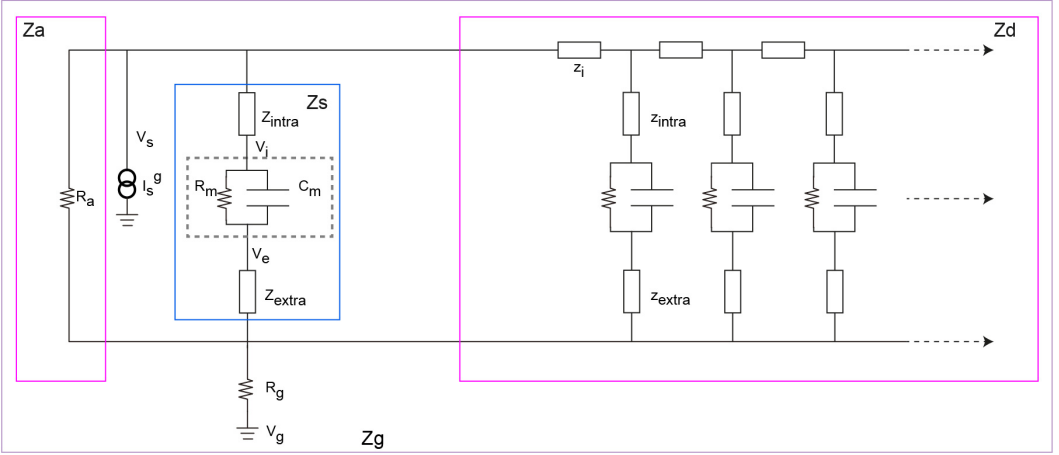

Figure 1: Method used for macroscopic impedance measurements and numerical simulations in different preparations. (A) Scheme of the experimental set-up. Left: Two borosilicate micropipettes with silver-chloride electrodes and a ground electrode are placed in the ACSF solution. One electrode is used in whole-cell configuration to send white-noise current and simultaneously measure the voltage response of the recorded neuron (acquisition sampling rate: $50 \mathrm{kHz}$ ); the other electrode used as a reference is located 5-10 $\mu \mathrm{m}$ away from the recorded neuron. Middle: Frozen Gaussian white noise (amplitude: \pm 5 to $20 \mathrm{pA}$; duration: $20 \mathrm{sec}$ ) is injected repeatedly into patched neurons. Right: Fourier modulus of the injected current as a function of frequency confirms that each frequency from 0 to $10000 \mathrm{~Hz}$ is uniformly sampled. (B) Equivalent electrical circuit between intracellular and extracellular electrodes. (C) Equivalent electrical circuit between the extracellular electrode and the ground. $Z_{a}$ is the impedance of the region defined by the isopotential surface $\mathcal{S}_{1}$ passing through the extracellular electrode and the first isopotential surface $\mathcal{S}_{g}$ that totally includes the neuron, $Z_{s}$ is the impedance of the part of the soma included between $\mathcal{S}_{1}$ and $\mathcal{S}_{g}, Z_{d}$ is the input impedance of the dendrite between surfaces $\mathcal{S}_{1}, \mathcal{S}_{g}$, and $Z_{g}$ is the impedance of the region between $\mathcal{S}_{g}$ and the ground. $Z_{g}=\left(Z_{a}\left\|Z_{s}\right\| Z_{d}\right) \oplus R_{g}$ where $\|$ means "in parallel" and $\oplus$ "in series". 
via the recording patch-clamp electrode in current-clamp mode. A quantity of 20 seconds of Gaussian white noise with zero mean and 5, 10 or 20 pA variance was injected. For each cell, we injected up to 71 times the same sequence of white noise (Fig. 1A). For each neuron, we computed the IV-curve by applying hyperpolarizing current steps of different intensities in current-clamp. Neurons for which the recording voltage of the responses was not inside the linear region of the IV-curve were excluded. For each trial, we calculated as a function of the frequency the modulus and Fourier phase of the voltage difference between the intracellular recording and extracellular reference electrode as well between the extracellular reference and ground electrode. We then averaged these measures to obtain a Fourier spectrum for each cell.

It is important to note that the numerical measurement of the phase induces a small delay $\Delta t$ between the real and digitally measured voltage, and this delay depends on the sampling frequency. This delay does not affect the modulus of the impedance, but is proportional to frequency. We have measured and corrected for this effect using the measurement of the resistance between the two electrodes in ACSF. Without this correction, we would have $\Phi(\nu)=0$ because saline is resistive for $\nu$ between 0 and $10 \mathrm{kHz}$. With the delay, we have $\Phi(\nu)=-\frac{0.5}{\nu_{e}} \nu$ where $\nu_{e}$ is the sampling frequency.

\subsection{Mathematical and Physical models used to simulate the experimental data}

To model the experimental data, we use Maxwell equations under the electric quasistatic approximation ${ }^{1}$ which was formulated in mean-field in previous studies $[12,19]$. At the first-order of this mean-field theory, the macroscopic impedance (in Fourier frequency space) of a point neuron in a heterogeneous medium is given by:

$$
Z(\omega)=\frac{V(\omega)}{I^{g}(\omega)}=\frac{R_{m}}{1+i \omega \tau_{m}}+\frac{A_{w}}{1+\sqrt{i \frac{\omega}{\omega_{w T}}}}+R_{a s y m p}+F(\omega)
$$

where $A_{w}=A_{o}+\frac{B_{o}}{1+i \omega \tau_{m w}}$. This expression was written by expliciting the following physical phenomena. The first right-hand term corresponds to the usual $\mathrm{R}-\mathrm{C}$ circuit of the membrane, the second term is due to ionic diffusion (in a linear approximation) as well as to electric polarization (if the polarization relaxation time is negligible). The last term takes into account other physical phenomena which may introduce a frequency dependence in the extracellular medium (such as capacitive effects ${ }^{2}$ ). The

\footnotetext{
${ }^{1}$ The electric quasistatic approximation consists of neglecting electromagnetic induction such that we have $\nabla \times \vec{E}=0$. It is important to note that in this approximation, the displacement current is taken into account and accumulation of charges can occur. Also note that there exists another approximation, the magnetic quasistatic approximation, where electromagnetic induction is not neglected [20].

${ }^{2}$ In principle, $F(\omega)$ accounts for other physical phenomena (such as electromagnetic induction, electric viscosity, etc) but these phenomena seem to have a negligible impact on the impedance
} 
physical meaning of the parameters is as follows: $R_{m}$ is the macroscopic membrane resistance, $\tau_{m}=R_{m} C_{m}$ is the membrane time constant, $A_{\omega}$ is the amplitude of the diffusive impedance (which is a real number when the polarization relaxation time (Maxwell-Wagner time $\tau_{M W}$ ) is negligible, $R_{\text {asymp }}$ is the asymptotic resistance for very high frequencies, and $\nu_{w T}=\omega_{w T} / 2 \pi$ is its Warburg threshold frequency (for details, see Supplemental Information, Appendix C).

The model used to take into account the effect of dendrites is the same as used previously [9], and consists of a model with a soma and a dendrite. Because we have a monopolar current source (electrode injection), the electrotonic length of a dendritic branch is given by:

$$
\lambda=\sqrt{\frac{r_{m}}{z_{i}}\left[1+\frac{z_{e}^{(m)}}{r_{m}}\left(1+i \omega \tau_{m}\right)\right]}
$$

where the following quantities are defined in the generalized cable: $z_{i}$ is the cytoplasm impedance per unit length, $r_{m}$ is the specific membrane resistance, and $z_{e}^{(m)}$ is the specific input impedance of the extracellular medium as seen by the membrane.

We have $z_{e}^{(m)}=z_{\text {intra }}+z_{\text {extra }}$ (from Fig. 1). The physical link between the generalized cable is the following. The current density in the dendritic stick has a component in parallel to the axis of the stick, and a component perpendicular to it. However, the electric conductivity is different for these two components. $z_{i}$ is associated to the parallel component, where the current density is physically related to the cytoplasm, and $z_{\text {intra }}$ is associated to the perpendicular component, which is physically related to the Debye layers in the inner side of the membrane. We have

$$
\left\{\begin{array}{l}
\frac{V_{m}}{I^{g}}=\frac{\lambda z_{i}}{\kappa} \operatorname{coth}\left(\frac{\kappa l}{\lambda}\right) \\
\frac{V_{i}}{I^{g}}=\frac{Z_{a} Z_{s} Z_{d}}{Z_{a} Z_{s}+Z_{s} Z_{d}+Z_{d} Z_{a}}+R_{g}
\end{array}\right.
$$

where the neuronal cable model in an "open configuration" (i.e, without return current) was used, as defined in [21] (see this paper for details).

Because the current that goes from the neuron to the extracellular space separates into several parts, one part that flows through the soma membrane, and another part that flows in the dendrite, we have used several impedances (see Fig. 1): $Z_{a}$ : the impedance of the extracellular medium in contact with the isopotential surface $S$ (which touches the extracellular electrode and surrounds the intracellular electrode), $Z_{s}$ : the impedance of the current flowing through the soma membrane (in contact with this surface $S$ ), and $Z_{d}$ : the input impedance of the dendritic tree. When measuring the equivalent impedance, we have $\left(Z_{a}\left\|Z_{s}\right\| Z_{d}\right) \oplus R_{g}$, where $R_{g}$ is the impedance between the ground and the first isopotential surface which comprises the neuron (see details in Supplemental Information, Appendix A).

The electric potentials $V_{i}$ and $V_{e}$ are taken at the inside and outside borders of the membrane, respectively, at the level of the soma and relative to reference

values for the frequency range $<1000 \mathrm{~Hz}$ investigated here [7]. 
A1

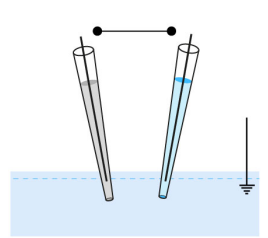

No cell

B1

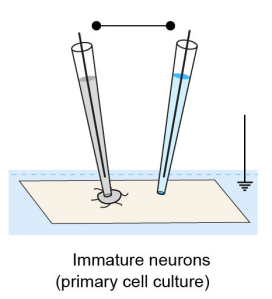

C1

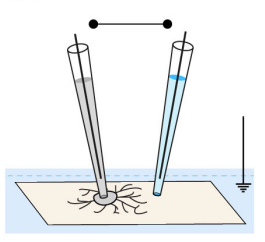

Mature neurons

(primary cell culture)

D1

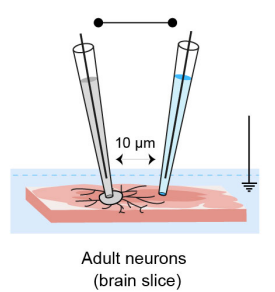

A2
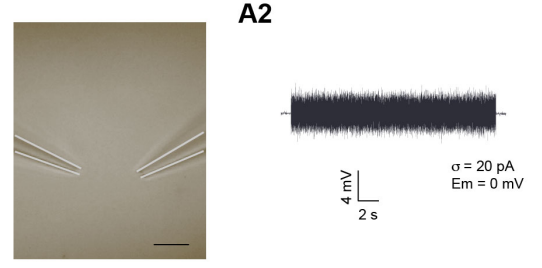

B2

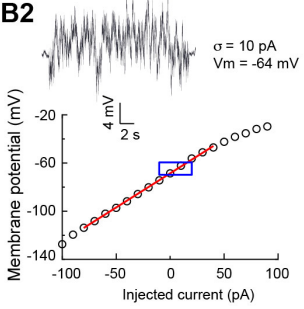

C2

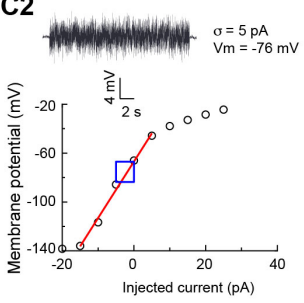

D2

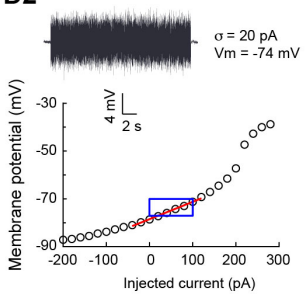

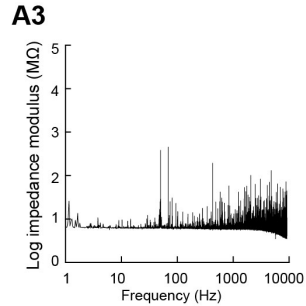
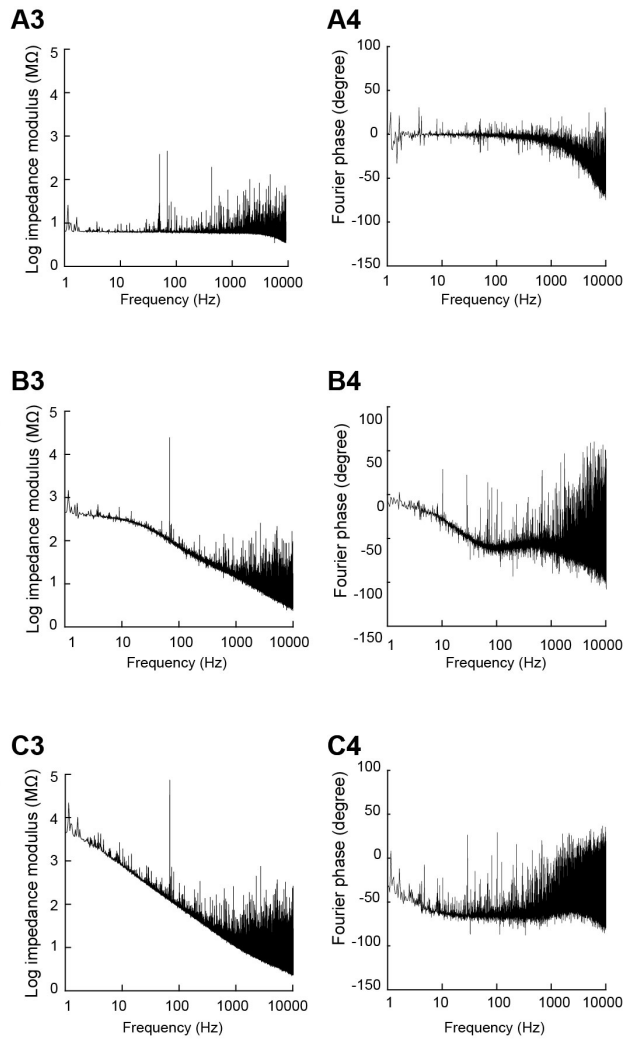

D3

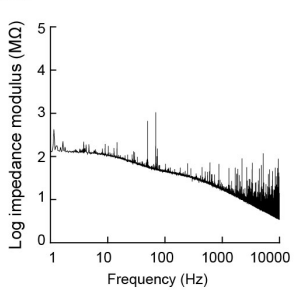

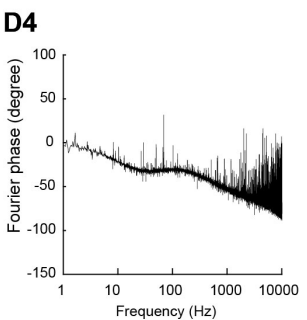

Figure 2: Impedance measurements in different preparations using a three electrode setup. (A) Measurement in ACSF. Description of the (1) experimental preparation (no biological sample is present in the ACSF solution) with (2) an example voltage response and for neuronal recordings, the corresponding I-V curve with the linear fit in red and the window of the voltage amplitudes during the current injection in blue. (3) and (4) show respectively the Fourier modulus and phase spectra of the voltage difference between whole-cell patch electrode and reference electrode as a function of frequency. B,C,D: same arrangement for different experimental preparations: recordings were made in primary cell cultures of striatal tissue (day 3 to day 10 after seeding) in which neurons with almost no neurites (B), with extended dendrites $(\mathbf{C})$ and from striatal neurons in acute horizontal brain slices $(\mathbf{D})$. 
point outside the neuron. According to the law of generalized current conservation, we have $\frac{V_{e}}{z_{e}^{(m)}}=\frac{V_{m}\left(1+i \omega \tau_{m}\right)}{r_{m}}$. Note that the parameters $R_{m}=r_{m} / \Delta S$ and $Z_{e}^{(m)}=$ $z_{e}^{(m)} / \Delta S$ (where $\Delta S$ is an element of surface area of the membrane) are macroscopic parameters, while $r_{m}$ and $z_{e}^{(m)}$ characterize the membrane at a microscopic level. This explains the difference between expressions $3 \mathrm{~b}$ and $3 \mathrm{a}$, because by definition we have $V_{i}=V m+V_{e}$. Note that if $\left|z_{e}^{(m)}\right|<<r_{m}$, then expression 3 b becomes equivalent to the input impedance of the dendrite (stick) in parallel with a portion of soma membrane (see Fig. 1).

$z_{e}^{(m)}$ is the specific input impedance of the extracellular medium, as sensed by the membrane, as also defined by the generalized cable theory [21]. This parameter can also be applied to the intracellular medium, in case of a current source from an intracellular electrode, to take into account the impedance of the intracellular medium between the tip of the electrode and the membrane. In the open configuration, $z_{e}^{(m)}$ can be a resistance for a resistive extracellular medium, but can be more complex when taking into account effects such as polarization, ionic diffusion, capacitive effects, etc. Thus, the form of this frequency dependence contains the contribution from the extracellular medium. Note that the expression in Eq. 2 shows that, in the open configuration, the electrotonic length depends on frequency, even for a resistive extracellular medium.

Also, in a "closed configuration" (where the neuron is electrically a closed system, where all currents loop back to the neuron), for a resistive medium, we have $z_{e}^{(m)}=$ $-\frac{r_{m} r_{e}}{\left(r_{i}+r_{e}\right)\left(1+i \omega \tau_{m}\right)}$ and $\lambda=\sqrt{\frac{r_{m}}{r_{i}+r_{e}}} \cdot z_{i}=r_{i}$ is the cytoplasm resistance per unit length and $r_{e}$ is the extracellular medium resistance per unit length, as defined in the RallTuckwell model. In this simplified and resistive model of the neurons, one assumes that the extracellular current flows parallel to the axis of the dendrite. In this case, we see that $\lambda$ does not depend on frequency. This is in accordance to the classic RallTuckwell cable theory [22, 23]. In contrast, in an open configuration with resistive media $\left(z_{e}^{(m)}\right.$ and $z_{i}$ are real positive), the modulus of $\lambda$ increases with frequency (see Eq. 3). Thus, in general, the electrotonic length $(|\lambda|)$ of a ball-and-stick neuron depends on frequency, which is not the case in the Rall-Tuckwell model, as shown above.

An interesting consequence of this is that, if one measures the impedance $z_{e}^{(m)}$ in a resistive configuration (for example in an isolated neuron embedded in ACSF), one should see the frequency dependence, which would validate the open configuration, as we will see in Results.

Finally, we fitted numerically the polar representation of the experimental data in Fourier frequency space, using the cubic spline method [24]. This method has a smooth derivative, which is appropriate for very noisy modulus and phase values. 
A1

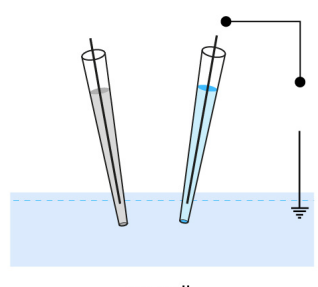

no cell

B1

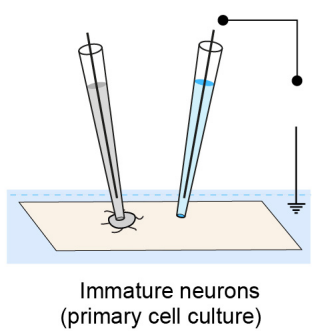

C1

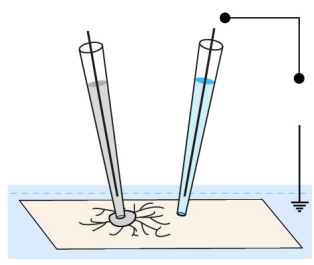

Mature neurons (primary cell culture)

D1

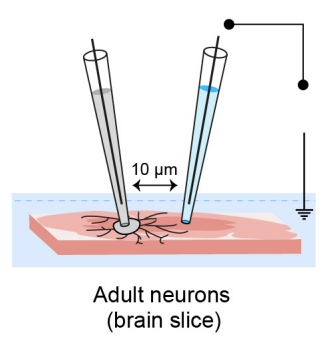

A2

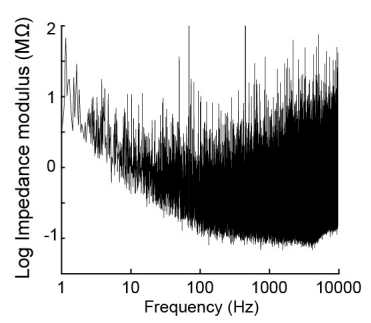

B2

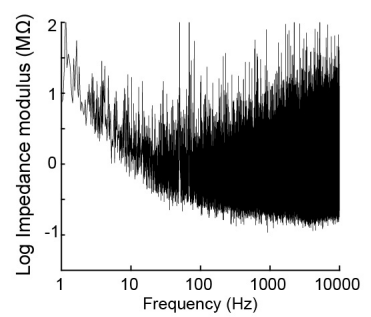

C2

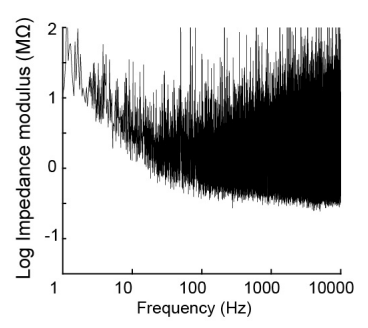

D2

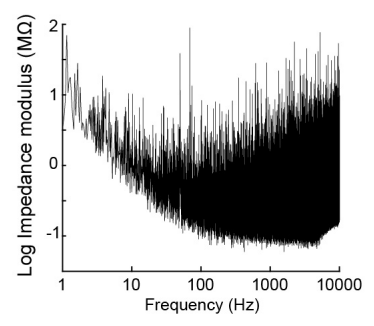

A3

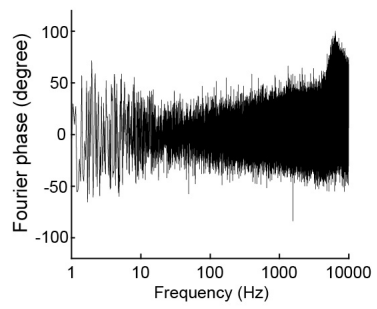

B3

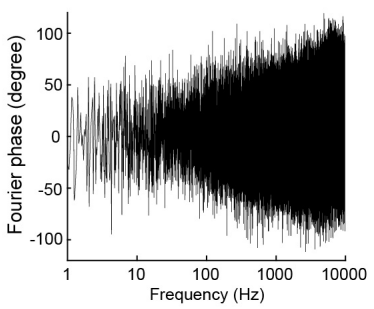

C3

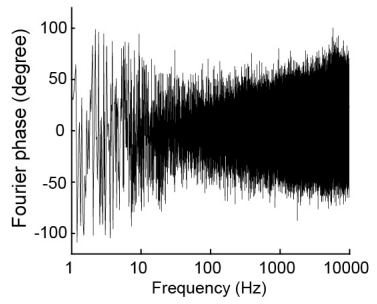

D3

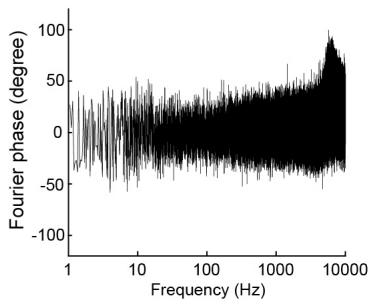

Figure 3: Measurements between the extracellular electrode and the ground (extracellular-to-ground impedance). Same arrangement of panels as in Fig. 2. Left: scheme of the recordings in the different preparations; Middle: modulus of the impedance; Right: phase of the impedance. 


\section{Results}

We first describe the experimental results and the different experimental configurations, then we propose different models to fit the experimental measurements.

\subsection{Experimental measurements}

The experimental protocol consisted of in vitro whole-cell patch-clamp recordings of striatal neurons, either from primary cell cultures or in acute brain slices, while simultaneously recording the potential in the vicinity $(5-10 \mu \mathrm{m})$ of the neuron using a second reference electrode (Fig. 1A, Fig. 2 and Fig. 3). The set-up thus consists of three electrodes: the intracellular electrode, the reference electrode and the ground. Frozen white Gaussian noise is injected in the cell via the patch-clamp electrode, and is measured according to two configurations: either one measures the intracellularto-extracellular (intracellular electrode with respect to reference electrode) potential, or the extracellular-to-ground (reference electrode with respect to ground).

Several templates (from 1 to 71 ) of the same Gaussian white noise with a flat spectrum between 0 and $10000 \mathrm{~Hz}$, were injected into each recorded neuron. Importantly, for the subthreshold range of voltage responses considered here $(-67 \mathrm{mV}$ $\pm 2.3 \mathrm{mV}$ ), the membrane I-V curve of striatal neurons was linear and only subthreshold responses to current injections $( \pm 5$ to $20 \mathrm{pA}$, adjusted according to the membrane resistance) were considered for analysis. More precisely, recordings, using the very same electrophysiological set-up, were obtained in four different experimental preparations (Figs. 2 and 3). We describe below the experimental results from the simplest experimental preparation in which two electrodes were added to a homogeneous ACSF solution without biological sample (Fig. 2A), to a more complex preparation, in which recordings were obtained from neurons within an acute brain slice (Fig. 2D). We also tested the influence of the dendritic arborization by recording either in non-arborized neurons with almost no neurites (Fig. 2B) or in arborized neurons with extended dendrites (Fig. 2C) in primary cell cultures. For each experiment, the Fourier modulus and phase of the voltage difference between the whole-cell recording electrode and the reference electrode were calculated and then averaged (as illustrated in Fig. 2). The same analysis was also applied to the voltage difference between the reference and ground electrodes (Fig. 3).

We describe below the experimental results from the simplest configuration of the two electrodes in ACSF, then neurons in a quasi-homogeneous medium ${ }^{3}$ (primary cell cultures), and finally a more complex configuration of neurons in acute brain slices.

\footnotetext{
${ }^{3}$ We refer to "quasi-homogeneous" the extracellular medium in culture, because it is very close to a homogeneous medium, and contrasts with the high heterogeneity seen in acute brain slices.
} 


\subsection{Measurements in ACSF}

In this section, we examine the simplest configuration consisting of two electrodes in a homogeneous medium solely constituted by ACSF, which corresponds to row A in Fig.2. The glass pipettes containing the electrodes were situated at a distance of 5 to $10 \mu \mathrm{m}$. Figure 4 shows the measured impedance between the two electrodes. The observed frequency dependence appears above $2 \mathrm{kHz}$ for the modulus (Fig. 4A) and above $500 \mathrm{~Hz}$ for the phase (Fig. 4B). This frequency dependence of the impedance modulus is negligible for frequencies lower than $2 \mathrm{kHz}$, but not for higher frequencies because $\Delta \log _{10} V \approx 50 \%$ between $2 \mathrm{kHz}$ and $10 \mathrm{kHz}$, similar to previous measurements using patch electrodes in the extracellular medium [6]. The phase of the impedance also shows a frequency dependence. It is negligible for frequencies smaller than $0.5 \mathrm{kHz}$ but for frequencies between $0.5 \mathrm{kHz}$ and $10 \mathrm{kHz}$, it varies of about 50 degrees. Our interpretation is that these frequency dependences come from the capacitive effect between the two electrodes (which was estimated of $C \approx 3.25 \mathrm{pF}$ ). We found that this capacitive effect is present in all experiments shown in the next sections, for intracellular and extracellular recordings.

It is important to note that, in this part of the experiments, we observe a linear phase lag $\Phi=-k \nu$ on the phase $\Phi$ of the impedance, as if electrode polarization had a non-negligible impact on the measurement. However, we observe that the constant $k$ is inversely proportional to the sampling frequency, which rules out a polarization effect (because with polarization, we would have $\Phi=-\left(k+k_{p}\right) \nu$ with $k_{p}$ independent of sampling frequency). Thus, the phenomenon of electrode polarization seems negligible in our experimental conditions, contrary to Miceli et al. [6] experiments. This difference probably occurs because we use smaller amplitude currents (about 20 pA here, compared to 175-500 pA in Miceli et al.). Note that the linear phase lag was removed in Fig. 4B.

According to Miceli et al. [6] and Wagner et al. [8], the electric conductivity of ACSF (called " $A C S F_{c}$ " in Miceli et al.) is similar to the conductivity measured between two points in the extracellular medium (around $0.5 \mathrm{~S} / \mathrm{m}$ ). We have not evaluated the value of this conductivity in the present experiments, because the very short distance between electrodes $(5-10 \mu \mathrm{m})$ induces capacitive effects. In this case, one would have to solve Laplace equation to estimate the conductivity from the macroscopic impedance measurement. In previous experiments such as Miceli et al. [6], the inter-electrode distance $(100-125 \mu \mathrm{m})$ is sufficiently large to avoid capacitive effects.

\subsection{Non-arborized neurons in primary cell culture}

In this section, we examine a relatively simplified system of immature neurons with little dendritic arborization, and laying in a simplified extracellular medium (primary cell culture). $\mathrm{n}=6$ neurons (with few or no dendrites) were recorded in this quasihomogeneous medium. This corresponds to row B in Fig.2. 


\section{A}

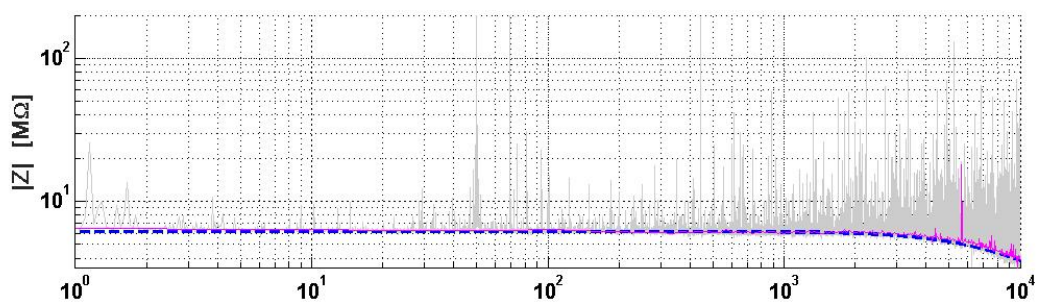

B

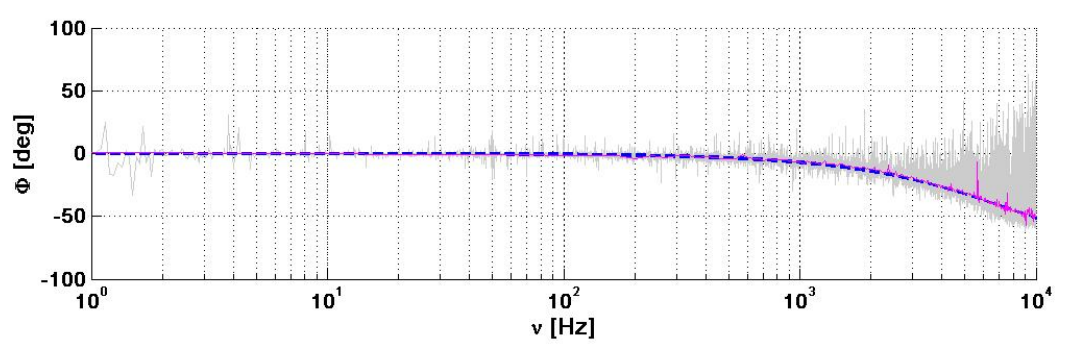

Figure 4: Impedance between two electrodes in ACSF. The distance between the two electrodes was of the order of 5 to $10 \mu \mathrm{m}$, The modulus (A) and the phase (B) are represented as a function of frequency $\nu$. A frequency dependence can be seen for $\nu>3 k H z(\mathrm{~A})$ and for $\nu>500 \mathrm{~Hz}(\mathrm{~B})$. The blue curve shows a capacitive effect of $3.25 \mathrm{pF}$. The magenta curve is a cubic spline fit of the experimental data (grey).
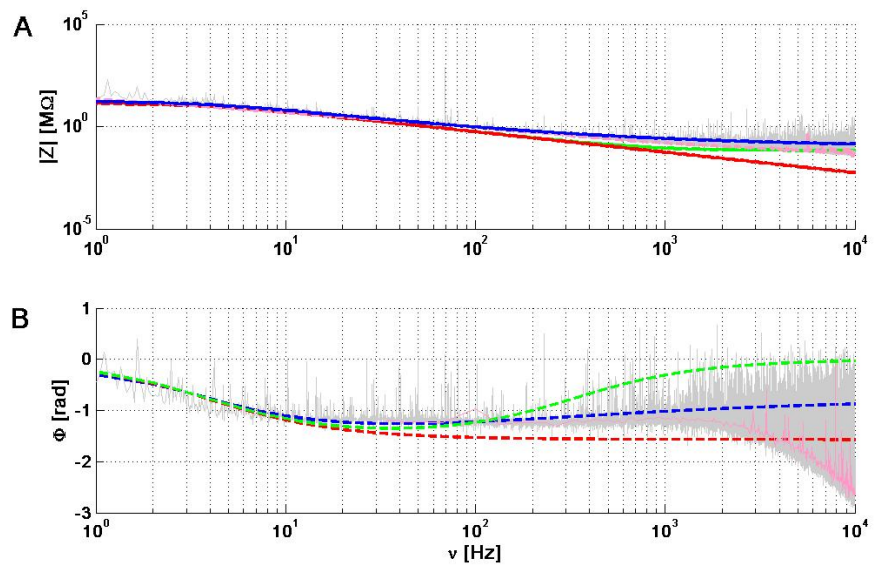

Figure 5: Cell-to-extracellular impedance $Z$ as a function of frequency $\nu$ for a nonarborized neuron (no dendrites) in a primary culture (quasi-homogeneous medium). A: modulus of $Z$ as a function of $\nu$. B: phase of $Z$ as a function of $\nu$. The different models tested were, Red: $\left(R_{m} \| C_{m}\right)$ model, Blue: $\left(R_{m} \| C_{m}\right) \oplus Z_{w} \oplus R_{a s y m p}$, Gray: experimental measurement, Green: $\left(R_{m} \| C_{m}\right) \oplus R_{\text {extra }}$ (see Supplemental Information, Appendix B). The symbol $\oplus$ means "in series with" and the symbol II means "in parallel with". Magenta: cubic spline fit of the experimental data. Parameters: $R_{m}=810 \mathrm{M} \Omega, \tau_{m}=30 \mathrm{~ms} ; A_{w}=495 \mathrm{M} \Omega, \nu_{w T}=0.1 \mathrm{~Hz}$, $R_{\text {extra }}=4 M \Omega$ and $R_{\text {asymp }} \approx 0.5 \mathrm{M} \Omega$. 
Figures 5 and 6 show the fitting of different models to experimental measurements obtained with isolated cells immersed in saline, which is a medium that can be considered as homogeneous and resistive as a first approximation. The fitting to these measurements shows that the intracellular-to-extracellular and extracellularto-ground macroscopic impedance clearly depends on frequency (Figs. 4 and 5).

The gray curve in Fig. 5 shows the measured impedance between the intracellular and extracellular electrodes. A RC circuit (membrane) in series with a resistance $R_{\text {medium }}$ (medium), modeling Region 1-2, cannot account for these impedance measurements (see green curves in Fig. 5). Simulating the impedance with different values of $R_{\text {medium }}$ (see Supplemental Information, Appendix B), could not mimic the experimental results. The red curve corresponds to $R_{\text {medium }}=0$, which is equivalent to consider that the membrane impedance is much larger than that of the extracellular medium. In this case also, it was not possible to properly fit the measurements.

The magenta curves in Fig. 5 are cubic spline fits of the experimental data. We observed a capacitive effect between the two intracellular and extracellular electrodes, as observed in ACSF, but is larger. This indicates that the mean electric permittivity of the intracellular medium in Region 1-2 is larger than that of ACSF. This capacitive effect is responsible for a steep decrease of the phase values at high frequencies. This is consistent with a diffusive effect, because a resistive model with membrane would have a phase around $\pi / 2 \mathrm{rad}$. for $\nu>100 \mathrm{~Hz}$, which is difficult to reconcile with this phase measurement.

The gray curve in Fig. 6 shows the measured impedance between the extracellular electrode and the ground. A model with a simple dendrite (stick) with resistive intracellular and extracellular media could not account for these experimental measurements. The red curve corresponds to an extracellular medium with negligible resistivity compared to that of the membrane $\left(z_{e}^{(m)} \approx 0\right)$. This case is equivalent to applying expression 3a. The green curve is such that $z_{e}^{(m)}=0.5 r_{m} \neq 0$.

In contrast, for the intracellular-extracellular measurement, we observed that the diffusive model $\left(F(\omega)=0\right.$ and $A_{w} \in \mathbb{R}$; see Eq. 1) simulates (with some error) these experimental measurements with a macroscopic $\mathrm{RC}$ circuit in series with a resistance and a diffusive impedance (see blue curve in Fig. 5). However, the residual error between experimental measurements and the diffusive model is very similar to what we observed for two electrodes in the ACSF (see Fig. 4). This model also fits the extracellular-to-ground measurement, despite the noise on the phase, for a stick where both intracellular and extracellular Debye layers are modeled with a diffusive model (blue curve in Fig. 6). We propose an interpretation of these results below.

Nevertheless, before any interpretation of the experimental results, it is important to note that we have fitted the models on the experimentally-measured phase values such as to minimize the mean square distance between experimental and theoretical values. Because there can exist several minima, we have completed the fit by constraining the optimal curve to fit either the minimal or maximal of the modulus envelope. To do this, we have computed the average values on small intervals 

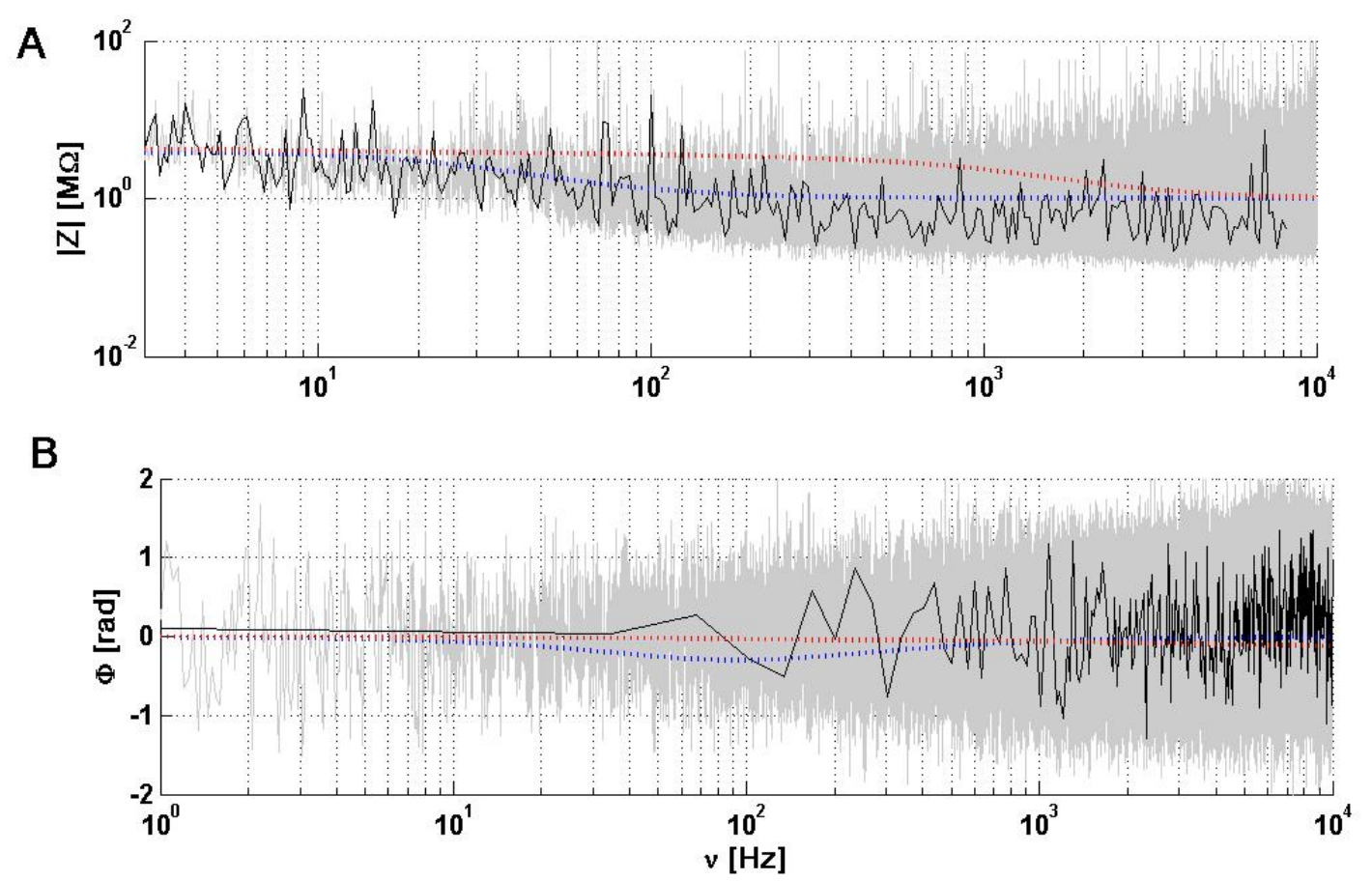

Figure 6: Extracellular-to-ground impedance for a non-arborized neuron in culture. A: modulus of the impedance as a function of frequency. B: phase of the impedance as a function of frequency. Dotted lines correspond to the mathematical model given by Eq. 3b (for details, see Fig. 7 and Supplemental Information, Appendix A). The different fits shown are: Blue: NMD - Membrane + diffusive intracellular and extracellular media ; Red: NMR - Neuron in a non-negligible resistive medium $z_{e}^{(m)} \neq 0$; Gray: experimental measurement; Black: For A, cubic spline of the logarithm of the modulus and frequency of experimental data; For B: cubic spline of the phase and logarithm of frequency. Parameters: in all models, $R_{m}=10 \mathrm{M} \Omega$ $\tau_{m}=25 \mathrm{~ms}, r_{d}=2 \mu \mathrm{m}, l_{d}=100 \mu \mathrm{m}$ and $Z_{g}=.1 \mathrm{M} \Omega ; \mathbf{N M R}: \sigma_{e}^{i}=5 \mathrm{~S} / \mathrm{m}$, $\frac{z_{m}^{(e)}}{r_{m}}=0.5, \mathrm{NMD}: \nu_{w T}=0.01 \mathrm{~Hz}, \frac{z_{e}^{(m)}}{r_{m}}=0.5+\frac{0.5}{1+\sqrt{i \frac{\omega}{\omega_{w T}}}}, \pi r_{d}^{2} \cdot z_{i}=\frac{1}{\sigma_{e}^{i}}+\frac{1}{1+\sqrt{i \frac{\omega}{\omega_{w T}}}}$.

of frequency $(\approx 1 \mathrm{~Hz})$ in the impedance modulus of the experimental data in each example. We have then adjusted the parameters of the different models to minimize the largest absolute distance between the modulus over the ensemble of intervals defined as above, between the average experimental and model values. This choice simplifies the comparison between the different models and experimental results. It is also important to note that log-log graphs give the false impression that there is more noise at high frequencies, but this is due to the high density of points.

\section{Analysis of the experiments}

We analyze these experiments according to the electrical configuration as depicted in Fig. 7. The isopotential surfaces indicated are central to our analysis. $S_{i}$ is the 
isopotential surface which corresponds to the potential measured at point $i$, where $S_{1}$ corresponds to the potential measured by the intracellular electrode, $S_{2}$ corresponds to the potential measured by the extracellular electrode and $S_{3}$ corresponds to the ground potential. For simplicity, we will call Region $i-j$ the domain delimited by surfaces $S_{i}$ and $S_{j}$.

First, because the extracellular medium surrounding the cell is mostly homogeneous and resistive, its macroscopic impedance cannot depend on frequency $[10]^{4}$. We thus conclude that the surface $S_{2}$ does not totally include the neuron, but cuts part of the soma, such that we have a portion of membrane over Region 2-3. Indeed, if the neuron was completely included into Region 1-2, then Region 2-3 would only consist of extracellular fluid, and would not exhibit (or exhibit negligible) frequency dependence, which was not what was observed (Fig. 6). This is in agreement with the fact that the intracellular and extracellular electrodes are very close to eachother (between 5 and $10 \mu \mathrm{m}$ ), compared to the size of the soma (estimated around $15 \mu \mathrm{m})$.

Second, the graph of the modulus of the impedance as a function of frequency in Region 1-2 (Fig. 5) is very different from Region 2-3 (Fig. 6). We thus conclude that the physical model is similar to the one represented in Fig. 7. Because the two points 1 and 2 are very close to each-other, we can simulate the impedance of Region 1-2 by an $\mathrm{RC}$ circuit in series with a diffusive impedance. However, the equivalent circuit of Region 2-3 cannot be the same because the current of the intracellular electrode divides into three parts: $I_{\text {intra }}^{g}=I_{a}^{g}+I_{s}^{g}+I_{d}^{g}$, where $I_{s}^{g}+I_{d}^{g}$ flows through the soma membrane and the dendrite, to the extracellular medium, while $I_{a}^{g}$ directly goes to the extracellular medium. Thus, the impedance of Region 23 is equivalent to expression $3 b$ (Supplemental Information, Appendix A). Because this equivalent impedance is much smaller than the input impedance of the stick and of the soma membrane, the extracellular-to-ground impedance is also much smaller than the intracellular-to-extracellular impedance. Despite this small value, the frequency dependence of the modulus of this impedance can be clearly seen (see Fig. 6). The experimental measurement of the impedance of Region 1-2 shows an additional capacitive effect similar to that seen in the previous section between intracellular and extracellular electrodes. However, this capacitive effect is negligible for the impedance measured in Region 2-3, which is coherent with the fact that the distance between the extracellular electrode and the ground is much larger than the distance between intracellular and extracellular electrodes.

Third, the growth of the modulus of the impedance of Region 2-3 for frequencies above $20 \mathrm{~Hz}$ is in full agreement with the diffusive model, as in Eq. 3b. This is

\footnotetext{
${ }^{4}$ Note that in [10], we have shown that if there is a strong spatial dependence of the electric parameters, a frequency dependence of the impedance appears. The equation used is equivalent to the generalized current conservation law. The latter can be written as $\nabla \cdot \vec{j}^{g}=0$ where $\vec{j}^{g}=-\left(\sigma_{e}+i \omega \varepsilon \nabla V\right)$, and we have$$
\nabla \cdot \vec{j}^{g}=\left(\sigma_{e}+i \omega \varepsilon\right) \nabla^{2} V+\nabla\left(\sigma_{e}+i \omega \varepsilon\right) \cdot \nabla V=0,
$$

which is equivalent to [10].
} 


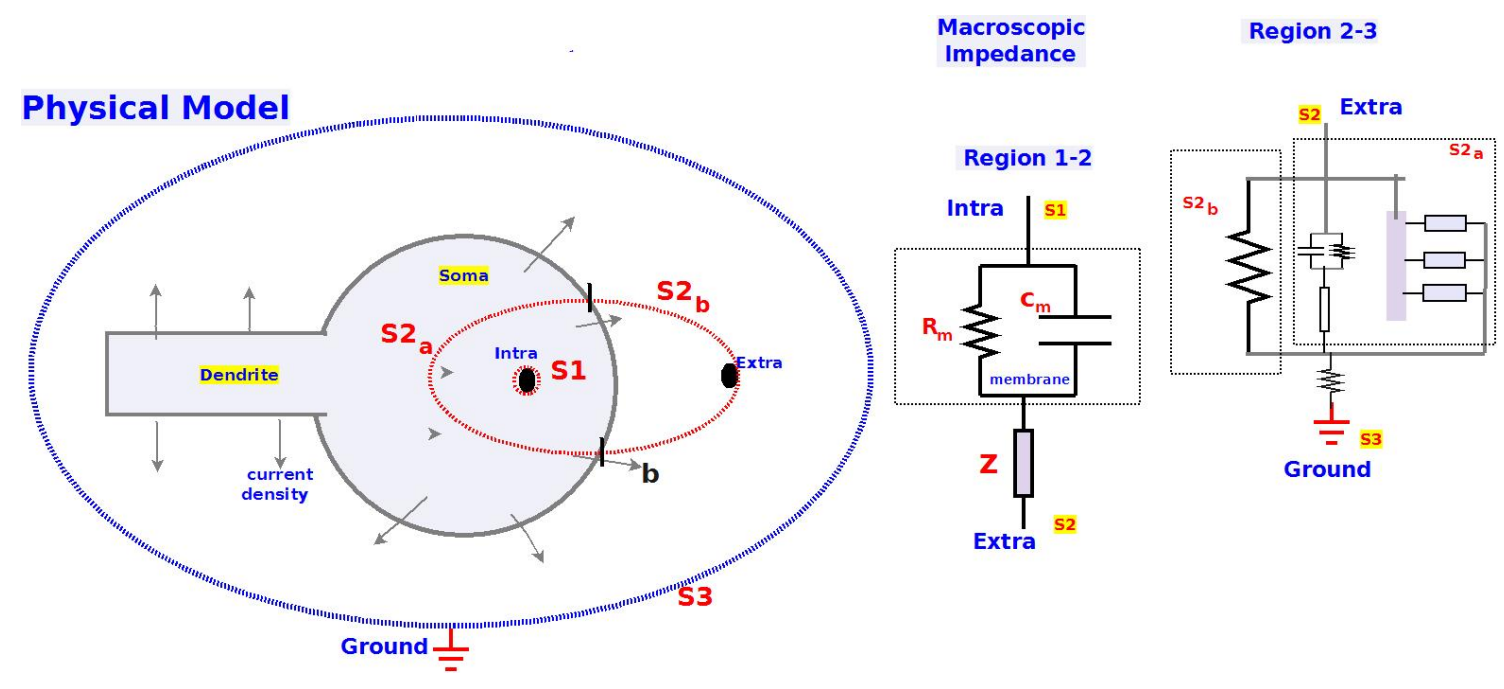

Figure 7: Electrical configuration of the cell and electrodes. Left: Physical model. The cell is schematized in gray, and the isopotential surfaces are labeled as $S_{1}$, $S_{2}, S_{3}$. Right: equivalent electric circuits for the impedances calculated between the intracellular and extracellular electrodes, as well as between extracellular and ground electrodes. This corresponds to the isopotential surfaces $S_{1}-S_{2}$ (Region 1-2) and $S_{2}-S_{3}$ (Region 2-3), respectively. $S 2_{b}$ is the part of surface $S 2$ where the current directly goes to the extracellular medium, whereas $S 2_{a}$ is the part of $S 2$ where the current that passes through the intracellular medium before going extracellular (soma + dendrite). In other words, in Region 2-3, we have taken Eq. 3b where $Z_{g}$ is a resistance. $R_{g}$ is the resistance between the ground and the first isopotential surface which includes the neuron (for more details, see Supplemental Information, Appendix A).

not the case with the other models considered here. The minimum of the modulus is directly linked to the membrane time constant. The longer the time constant, the higher the frequency of the minimum. We estimate from the position of the minimum, a membrane time constant of about $30 \mathrm{~ms}$, which is consistent with the membrane time constant estimated in this configuration. The growth of the modulus shows that $\frac{z_{e}^{(m)}}{r_{m}}$ cannot be neglected (Fig. 6). The value of this ratio is estimated between 0.5 and 1 for null frequency (Supplemental Information, Appendix D).

Fourth, the values of the ratio $\alpha=R_{m} / A_{w}$ (model NmD for $\nu=0$ ) are approximately $\alpha \approx 1-2$ for both Region 1-2 and Region 2-3. Here, NmD stands for a model where each differential element of membrane is equivalent to a parallel $\mathrm{RC}$ circuit and where the intracellular and extracellular media are diffusive. These ratios do not correspond to a singular case, but were similar in all cells examined (Supplemental Information, Appendix $\mathrm{G}$ ) in the same experimental conditions. Because neglecting $A_{w}$ would amount to have the NMR model of Fig. 6, these experimental results show that the intracellular and external Debye layers cannot be assimilated to a simple resistance. In contrast to $\mathrm{NmD}$, the $\mathrm{NmR}$ configuration has resistive intracellular and intracellular media, but with the same membrane impedance. Moreover, the 
threshold frequency cannot be considered as infinitely large $\left(\nu_{w T}=0.1 \mathrm{~Hz}\right.$ in Fig. 5 and $\nu_{w T}=0.001 \mathrm{~Hz}$ in Fig 6) because in this case, we would also have the NMR model. The threshold frequency $\nu_{w T}$ of the diffusive impedance between surfaces $S_{1}$ and $S_{2}$ is greater than that between surfaces $S_{2}$ and $S_{3}$. This shows that surfaces $S_{1}$ and $S_{2}$ have very different curvatures. The small value of the Warburg threshold frequency $\left(\nu_{w T}=\omega_{w T} / 2 \pi\right)$ indicates that the curvature radius of the isopotential surface $S_{2}$ is much larger than the surface $S_{1}$, which is consistent with the fact that surface $S_{1}$ is at a zero distance from the tip of the intracellular electrode, which is not the case for surface $S_{2}$ (Fig. 6; for more details, see Supplemental Information, Appendix D).

Fifth, even for zero frequency, one does not observe the typical resistance of $A C S F$. If we calculate the resistance sensed by a fictive spherical source of diameter equivalent to the intracellular electrode $(\sim 1 \mu \mathrm{m})$, embedded in $A C S F_{c}$ with an electric conductivity of $\sigma_{e}=0.5 \mathrm{~S} / \mathrm{m}$ [6], we have $\approx \frac{1}{4 \pi \sigma_{e}}\left[\frac{1}{r_{1}}-\frac{1}{r_{2}}\right] \approx 40 \mathrm{k} \Omega$. Here, $r_{1}$ is the diameter of the fictive source, and $r_{2}$ is the average distance between the membrane and the spherical source. This value of $4 \mathrm{k} \Omega$ is smaller than the value in Region 1-2 predicted by the diffusive model for a near-zero frequency, because this value is much smaller than the estimated $A_{w}$, which is about $30 \%$ to $45 \%$ of that of the membrane. This result is surprising, and different from the approximation usually made, assuming that the intracellular impedance is much smaller in amplitude compared to the membrane impedance for near-zero frequencies.

How to explain this result? According to the theory and experimental measurements of the electric conductivity of heterogeneous media [26, 27], the phenomenon of polarization occurs when an electric field is applied, and this contributes to lower the electric conductivity of the medium. Similar considerations apply to the phenomenon of ionic diffusion, which also lowers the conductivity [9, 21]. In the polarization effect, the electric field polarizes the intracellular medium, which determines an electric resistivity that is larger than the most conductive part of the medium. Thus, electric polarization diminishes the apparent electric conductivity in a heterogeneous medium, which directly affects the value of $A_{w}$ (Supplemental Information, Appendix C). Nevertheless, the present measurements show that the characteristic relaxation time of polarization (also called Maxwell-Wagner time), is negligible in these experimental conditions because the diffusive model alone can fit very well the experimental results ${ }^{5}$. If the polarization relaxation time was not negligible, there would be an additional frequency dependence to take into account in addition to the diffusive model. For example, if we take the diffusive model with polarization, and a non-negligible relaxation time, then we would have a supplementary frequency dependence relative to the diffusive model (not shown). Note that the diffusive model is equivalent to a model where the polarization relaxation time becomes very large when the frequency tends to zero (Supplemental Information, Appendix F). Because the diffusive model fits very well the measurements, adding polarization and or an additional resistance is not necessary. We conclude that the intracellular medium

\footnotetext{
${ }^{5}$ Note that the diffusive model stems from the application of quasi-static statistical thermodynamics to the model of Gouy-Chapmann-Stern-Debye [19, 28].
} 
is such that the cutoff frequency $f_{c}=1 / 2 \pi \tau_{m w}$ of the low-pass filter due to electric polarization [11] is larger than $10 \mathrm{kHz}$ in this experiment.

The large extracellular medium impedance can also be explained by the tortuous structure of the intracellular medium. Because the electric field lines cannot follow this tortuous structure, the charges that are moving in the extracellular space due to the electric field are subject to various obstacles [39]. To move away from these obstacles, ionic diffusion is necessary in addition to the electric field. Thus, it is expected that the impedance modulus of such a tortuous medium is larger than ACSF.

The high value of the impedance (high value of $A_{w}$ ) is also due to the fact that the size of the soma is much larger than the plasmic membrane thickness. The ratio between the two is of the order of 1000 , because the membrane has a thickness around $7.5 \mathrm{~nm}$ while the soma has a typical size around $10 \mu \mathrm{m}$. If we consider a slice inside the soma, we get approximately $R_{\text {soma internl }}<A_{w} / 1000=4 M \Omega / 1000 \approx 4 k \Omega$ which is much smaller than $R_{m}$. We can repeat this for further slices in series of similar thickness. Because $A_{w}$ contains the contribution of external and internal Debye layers ${ }^{6}$, the value of $R_{\text {soma internal }}$ evaluated is smaller than $4 k \Omega$. Thus, the medium inside the soma is much more conductive than the membrane, as postulated by the standard model, but the difference is not as large as assumed by that model (see red curve in Fig. 5).

Finally, we observed that the impedance measured between the extracellular electrode and the ground is much more noisy than the intracellular-to-extracellular impedance. However, it should be noted that, besides the presence of this higher level of noise, the measurements also show that the modulus of the two theoretical diffusive impedances in Regions 1-2 and 2-3 are in full agreement with the experimental results. This indicates that the extracellular electrode is sufficiently far away from the membrane and does not disturb its ionic environment (Debye layers). It is nevertheless close enough so that the isopotential surface $S_{2}$ only cuts a portion of the soma.

To conclude this analysis, the present measurements in non-arborized neurons in primary cell culture cannot be made compatible with a resistive system, both for the modulus and phase of the impedance. However, both seem compatible with the impedance profile predicted by a diffusive system, which we interpret as being essentially due to the presence of Debye layers surrounding the membrane. However, to be rigorous, we must also mention the influence of the multiple obstacles inside and outside the neuron, which also have Debye layers and will influence the current flow.

\footnotetext{
${ }^{6}$ By definition, the external (or internal) Debye layer is the region at the interface between the membrane and the extracellular (or intracellular) medium. It was estimated that the electric potential over this region attenuate by $e \approx 2.718$ times its value on the membrane surface. Its thickness is called the Debye length. For more details on the notion of Debye layers, see [28].
} 

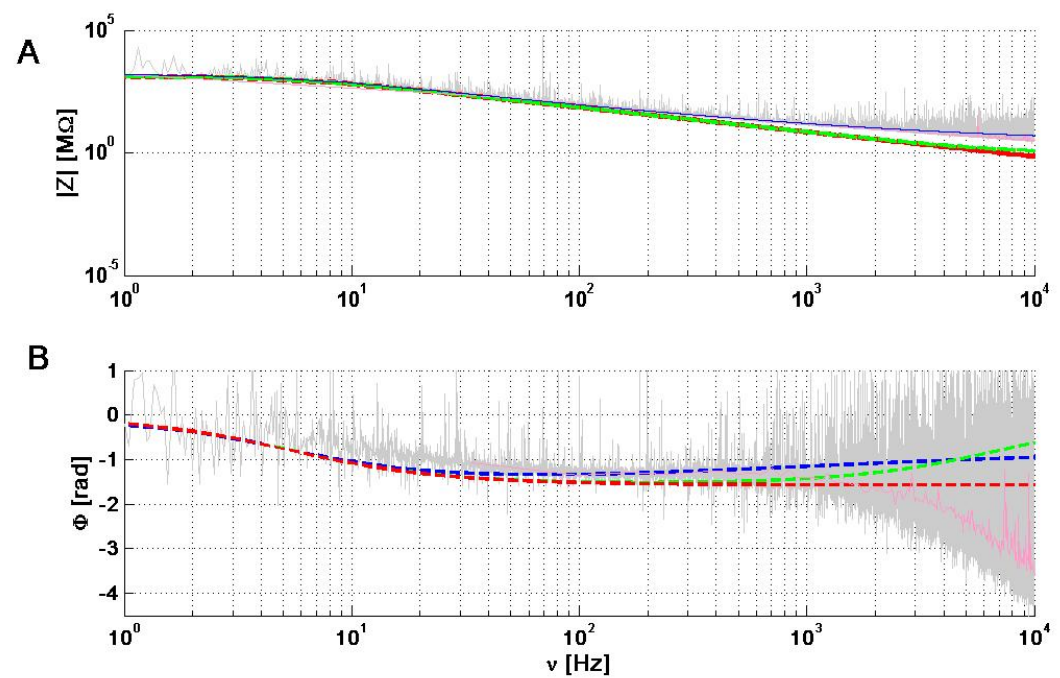

Figure 8: Intracellular-to-extracellular impedance $Z$ as a function of frequency $\nu$ for an arborized neuron in a primary cell culture (homogeneous medium). Magenta: cubic spline fit of the experimental data. Similar description as in Fig. 5 with parameters $R_{m}=1350 \mathrm{M} \Omega, \tau_{m}=28 \mathrm{~ms} ; A_{w}=450 \mathrm{M} \Omega, \tau_{m w} \approx 0 \mathrm{~s}, \nu_{W}=0.3 \mathrm{~Hz}$, $R_{\text {extra }}=1 M \Omega, R_{\text {asymp }}=1 M \Omega$.

\subsection{Arborized neurons in primary cell culture}

In this section, we followed the same approach as in the previous section, but in the case of recordings made in more mature neurons with extended dendritic arborization, in primary cell cultures $(n=6$ cells), which corresponds to row C in Fig. 2. Using the same scheme as in Fig. 7, we also investigate how the presence of dendrites influences the measured macroscopic impedance in this quasi-homogeneous medium.

By comparing the intracellular-to-extracellular impedances in Figs. 5 and 9, one can observe that the presence of dendrites has a negligible influence on the measured intracellular-to-extracellular impedance. The quality of the fit of the extracellularto-ground impedance in Fig. 10 is very similar to that of Fig. 6. We considered a stick radius of $2 \mu m$ (as in Section 3.3) and a stick length of $600 \mu m$ (instead of $100 \mu \mathrm{m}$ ) to take into account the presence of a more extended dendritic arborescence (Supplemental Information, Appendix E). Note that the values of radius and length of the stick are not unique because the simulations can fit the experimental results equally well with a large number of dendritic parameters. However, the area of the dendritic stick is fixed when we fix the ratio $z_{e}^{(m)} / r_{m}, r_{i}$ and $\tau_{m}$ (see Eqs 3 and Eq. 2; for more details, see [21]). Thus, keeping the same stick diameter as in previous section, the fact that the length of the stick is much larger than in immature neurons, indicates that, in these experiments, the presence of dendrites has a much larger impact on the extracellular-to-ground impedance compared to the previous 

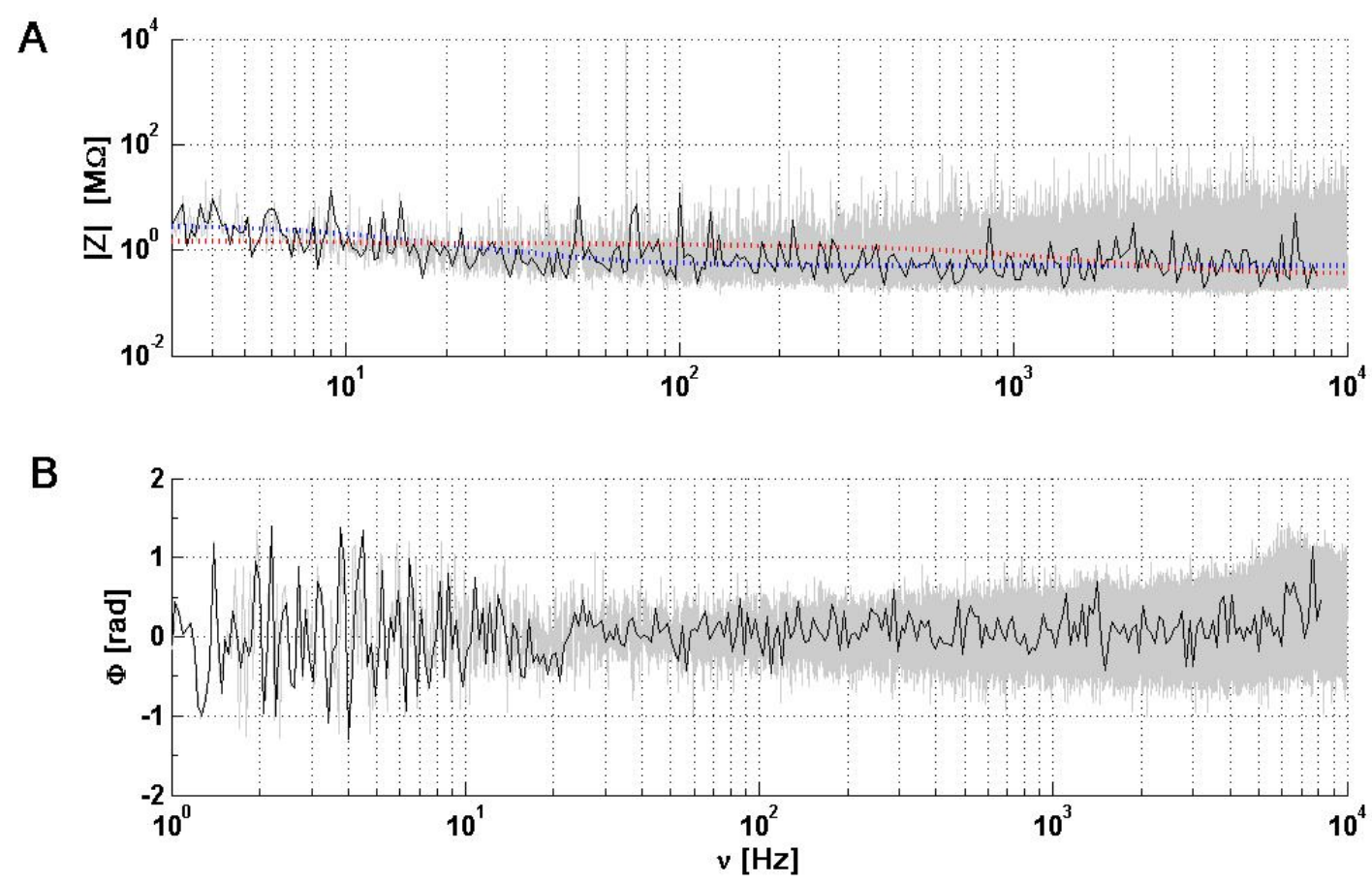

Figure 9: Extracellular-to-ground impedance for an arborized neuron in culture. A: modulus of the impedance as a function of frequency. B: phase of the impedance as a function of frequency. Same color code as in Fig. 6. Dotted lines correspond to the mathematical model given by Eq. 3b (for details, see Fig. 7 and Supplemental Information, Appendix A). Parameters: $r_{d}=2 \mu m l_{d}=600 \mu m, R_{m}=12 \mathrm{M} \Omega$ $\tau_{m}=35 \mathrm{~ms}, \sigma_{e}^{i}=5 \mathrm{~S} / \mathrm{m}$ and $Z_{g}=0.2 \mathrm{M} \Omega$. NMR: $\frac{z_{m}^{(e)}}{r_{m}}=.5 ; \mathbf{N M D}: \nu_{w T}=0.1 \mathrm{~Hz}$, $\frac{z_{e}^{(m)}}{r_{m}}=0.5+\frac{0.5}{1+\sqrt{\frac{i \omega}{\omega_{w T}}}}, \pi r_{d}^{2} \cdot z_{i}=\frac{1}{\sigma_{e}^{i}}+\frac{1}{1+\sqrt{\frac{i \omega}{\omega_{w T}}}}$.

section. We have used a dendrite length which is 6 times larger for arborized neurons, which corresponds to visual inspection, but the results were weakly dependent on the exact value of this parameter (not shown).

Thus, the fits of different theoretical models to experimental measurements of (arborized) neurons with dendrites give very similar results to those obtained in neurons without dendrites (non-arborized) examined in the previous section. In other words, the mean square error of the fit of the diffusive model is about 50 times smaller than that of the resistive model (with or without dendrites; see Supplemental Information, Appendix G). The same capacitive effect as in ACSF was observed here for both arborized and non-arborized neurons (Fig. 8 and Fig. 4 in Section 3.2 and Fig. 5 in Section 3.3). It is thus important to stress that the 3-point measurement used here allowed us to directly measure the physical effect of the presence of dendrites on experimental measurements. 

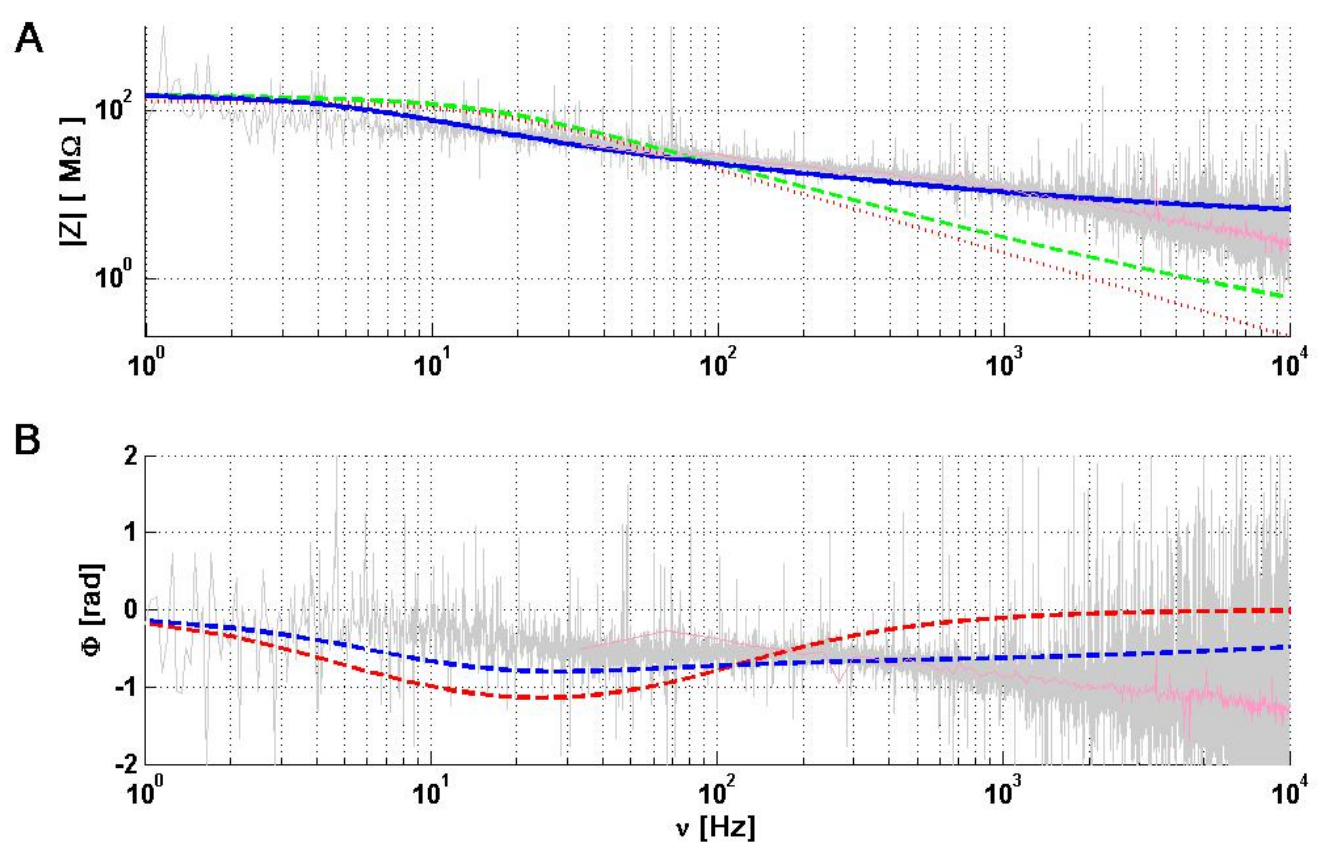

Figure 10: Intracellular-to-extracellular impedance $Z$ as a function of frequency $\nu$ for a neuron recorded in acute brain slice. A: modulus of $Z$ as a function of $\nu$. B: phase of $Z$ as a function of $\nu$. Parameters: $\tau_{m}=10 \mathrm{~ms}, R_{m}=128 \mathrm{M} \Omega$; $A_{w}^{\alpha}=60 M \Omega, \nu_{w T}^{\alpha}=0.5 \mathrm{~Hz}, R_{a s y m p}^{a}=6 M \Omega ; A_{w}^{\beta}=16 \mathrm{M} \Omega, \nu_{w T}^{\beta}=40 \mathrm{~Hz}$ and $R_{\text {asymp }}^{\beta}=6 M \Omega$. The color code is red : fit with resistive intracellular and extracellular media (16 M $\Omega$ ); green: fit with a diffusive intracellular medium (a) and a resistive extracellular medium $(16 \mathrm{M} \Omega)$; blue: fit diffusive intracellular and extracellular media (see Eq. 4); Magenta: cubic spline fit of the experimental data. The latter case best fits the experimental measurements. The mean square error of the diffusive model is about 3 -fold less that than of the resistive model (see Supplemental Information, Appendix G).

\subsection{Arborized neurons in acute brain slices}

In this section, we present and analyze impedance measurements of mature and fully-arborized neurons ( $\mathrm{n}=9$ cells) recorded in acute brain slices (row D in Fig. 2). In this case, the tissue surrounding the neuron is quasi-intact, and the extracellular medium is the neuropil, which is very heterogeneous [40]. We will now analyze the effect of such an environment on the frequency dependence of the macroscopic impedance.

We used the same experimental method (3 point recording) as in previous sections (see Fig. 7). We observe a different frequency dependence for the impedance of Region 1-2 (see Figs. 5, 8 and 10). This shows that the presence of a complex extracellular medium (neuropil) causes more complex effects in the vicinity of the neuronal membrane. However, if we consider two different threshold frequencies for 
intracellular and extracellular media, we obtain

$$
Z=\underbrace{\frac{R_{m}}{1+i \omega \tau_{m}}}_{\text {membrane }} \oplus \overbrace{\underbrace{\frac{\overbrace{w}^{\alpha}}{1+\sqrt{i \frac{\omega}{\omega_{w T}^{\alpha}}}} \oplus R_{\text {asymp }}^{\alpha}}_{\text {diffusive model }}}^{\text {intracellular medium }} \oplus \overbrace{\frac{A_{w}^{\beta}}{1+\sqrt{i \frac{\omega}{\omega_{w T}^{\beta}}}} \oplus R_{\text {asymp }}^{\beta}}^{\text {extracellular medium }}
$$

which gives excellent fits (Fig. 10). Note that we have kept the characteristics (parameters) of the previous experiments for modeling the intracellular medium.

In Region 2-3, we observed a similar frequency dependence as in previous configurations (see Figs. 6, 9 and 11). In the case of the diffusive model, we used a model of the macroscopic impedance $Z_{b}$ (impedance of the extracellular medium as sensed by Surface $S_{2}$; see Fig. 7), which was taken from a previous study [9], with the difference that we added a series resistance $(8 M \Omega)$. This addition is justified in Supplemental Information, Appendix C (see Eq. C.5). Note that this impedance is necessarily macroscopic because it is given by the ratio of the measured current between two points separated by macroscopic distances (as defined by the region in between the two isopotential surfaces going through each point). Accordingly, we used $Z_{b}=8+\frac{8}{1+\sqrt{\frac{i \omega}{w_{w T}}}} M \Omega$ where $\nu_{w T}=40 \mathrm{~Hz}$, as determined in [9]. Note that the particular choice of the parameters $\left(A_{w}=R_{a s y m p}=8 M\right.$ and $\left.w_{w T}\right)$ can be varied (by approximately $\pm 50 \%$ with no qualitative change in the results (see Supplemental Information, Appendix G). While many combinations of parameters fit the data for diffusive models, we were unable to find a single parameter set of resistive models that could fit the data.

The modulus of the extracellular impedance between the extracellular electrode and the ground (Region 2-3) in acute brain slices can be different from that in primary cell culture. The impedance sensed by the current source that does not flow through the dendrite and the cell membrane (Fig. 7) on Region 2-3 (between the extracellular electrode and the ground) are experimental conditions similar to extracellular measurements performed in other studies [5, 6]. Thus, for Region 2-3, we have chosen a similar model as for the experiments in primary cell culture.

\section{Analysis of the brain slice experiments}

In the acute brain slice preparation, the observations are similar to the observations in primary cell culture, except that the best fit is obtained here when we use different threshold frequencies for intracellular and extracellular media. We now focus on the analysis and interpretation of such different threshold frequencies.

According to Gomes et al. [9], the extracellular impedance in brain slices has a threshold frequency between 40 and $60 \mathrm{~Hz}$. Compared to the present experiments, the thickness of the extracellular medium was much larger in [9], because the extracellular electrode was located at a distance about 10 times larger. Indeed, in [9], the macroscopic impedance of the extracellular medium was larger than that of the 


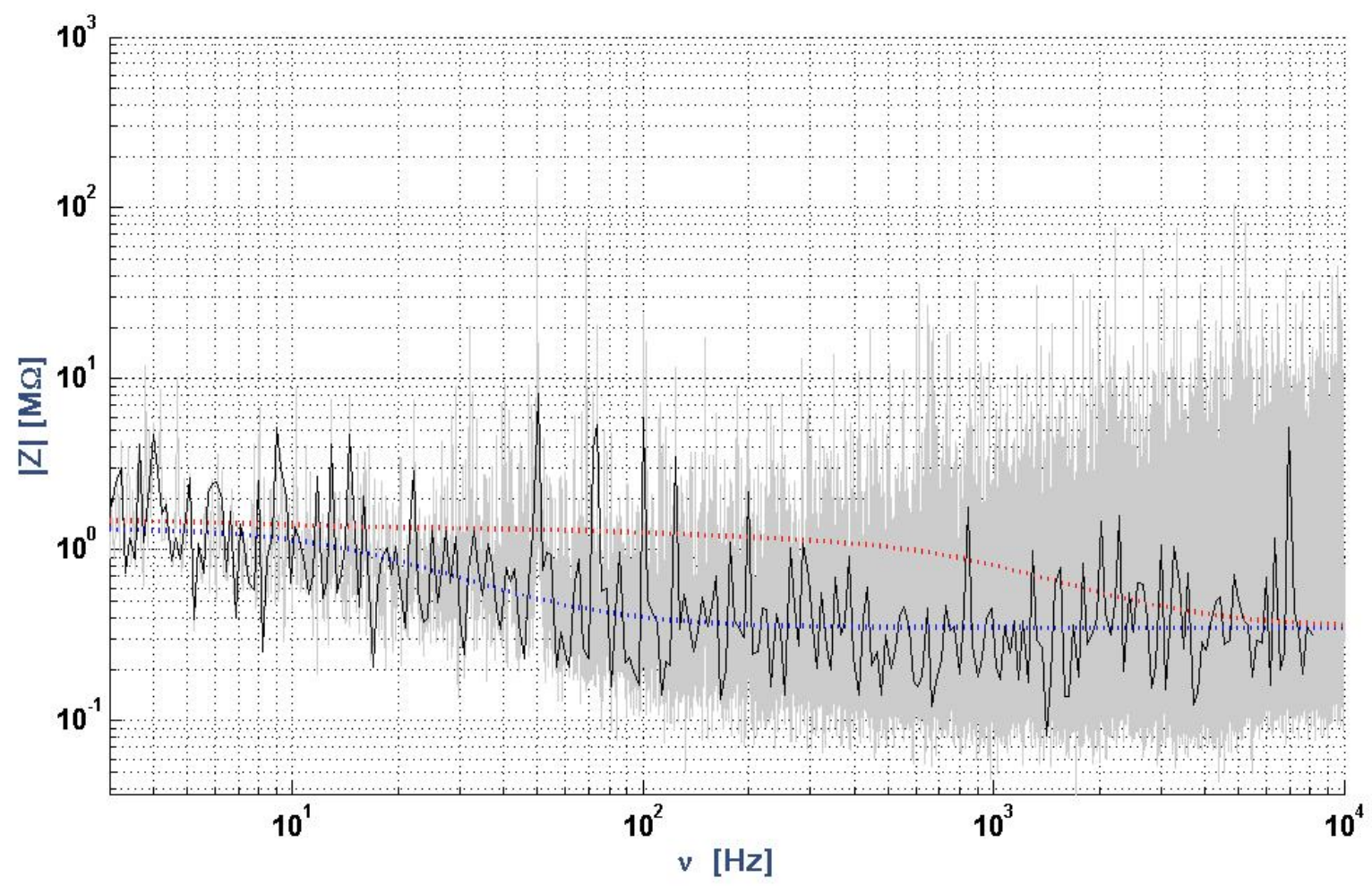

Figure 11: Extracellular-to-ground impedance for a neuron recorded in acute brain slice. The same color code as in Fig. 6 was used. Dotted lines correspond to the mathematical model given by Eq. 3b (for details, see Fig. 7 and Supplemental Information, Appendix A). The modulus of the impedance is represented as a function of frequency. Parameters: $R_{m}=5 M \Omega, \tau_{m}=10 \mathrm{~ms}, r_{d}=2 \mu m, l_{d}=600 \mu m$, $\sigma_{e}^{i}=5 \mathrm{~S} / \mathrm{m}$ and $Z_{g}=.2 \mathrm{M} \Omega$. NMR: $\frac{z_{m}^{(e)}}{r_{m}}=.5 ;$ NMD: $\nu_{w T}=0.01 \mathrm{~Hz}$, $\frac{z_{e}^{(m)}}{r_{m}}=0.5+\frac{0.25}{1+\sqrt{i \frac{\omega}{\omega_{w T}}}}, \pi r_{d}^{2} \cdot z_{i}=\frac{1}{\sigma_{e}^{i}}+\frac{1}{1+\sqrt{i \frac{\omega}{\omega_{w T}}}}$. 
intracellular medium. Thus, the present results, together with Gomes et al. [9], suggest that the threshold frequency of the extracellular medium is larger than that of the intracellular medium. This larger threshold may indicate that the medium is more tortuous inside the cell compared to the extracellular medium.

This result is also in qualitative agreement with the experimental measurements of Gabriel et al. [7] and Wagner et al. [8] because the apparent conductivity and permittivity of the diffusive model are respectively very low and very high, which gives a very large dielectric relaxation time (Supplemental Information, Appendix F). According to the experimental results of Gabriel et al., the electric permittivity of the extracellular medium is much larger in the neuropil, compared to that of the extracellular fluid in cultures. The value of the permittivity is in agreement with the experimental measurements $[7,8]$ and is estimated to be $10^{5}$ to $10^{7}$ times larger than that of ACSF. Notice that the results of Wagner et al. were obtained in vivo and are not identical to that of Gabriel et al. obtained in vitro. This difference may be due to the fact that the linear approximation of the medium impedance may be valid in vitro but not in vivo. Can the linear approximation be considered as a first estimate? Is the deviation due to the presence of ongoing (spike) activity? These questions await experimental testing.

\section{Discussion}

In this study, we provide for the first time, impedance measurements around neurons, in different experimental preparations and comparison between extracellular and intracellular impedance measurements. The aim was to determine which is the most plausible physical model that accounts for the electric properties of the extracellular medium. We used an experimental measurement consisting of three points, an intracellular electrode, an extracellular electrode in the close vicinity of the membrane (a few microns), and a reference electrode (ground) located far away. Using the same recording setup, we have considered different preparations ranging from acute brain slices, primary cell cultures with neurons of different morphological complexity, and measurements in ACSF solution as control. In addition to these measurements, we have provided a detailed fitting of the measured impedance using several biophysical models of the extracellular medium. The main conclusions are that (1) in no circumstance, the resistive model is able to fit the whole data set; (2) the measurements point to a dominant role of ionic diffusion, as soon as a membrane is present; (3) the measurements suggest that the underlying mechanism is the ionic diffusion associated to the Debye layers around the membrane; (4) additional capacitive effects may be needed to explain the differences between experimental preparations.

Regarding the first conclusion, a resistive medium cannot account for any of the measurements, except when electrodes are placed in ACSF. The resistive nature of the impedance measured in ACSF is expected because this saline solution is the simplest case of a resistive medium. In this case, we had to consider an additional 
capacitive effect in parallel with the two electrodes (see Section 3.2). This shows that the measurements with the micro-pipettes do not create an apparent frequencydependence of the measured medium, and once the capacitive effect is removed, one recovers the correct resistive measurement in this case.

The second conclusion, that ionic diffusion plays a prominent role, is supported by our fitting analysis considering different model alternatives. This suspected presence of ionic diffusion (and the associated Warburg impedance) agrees with previous studies showing a role for ionic diffusion. Macroscopic measurements of the impedance of brain tissue $[7,8]$ showed a frequency dependence of the electric parameters which is consistent with ionic diffusion, as pointed out by a theoretical study [19]. This study developed a mean-field formalism of Maxwell equations, which was necessary to properly account for macroscopic measurements that imply averages over large spatial volumes. In this mean-field framework, the predicted frequency scaling of ionic diffusion was found to be consistent with the experimental observations. It was further shown, using a two-electrode measurement setup with intracellular and extracellular electrodes, that ionic diffusion also accounted for the observed frequency dependence [9]. However, as pointed out in [16], the dendrites were neglected in our previous study. We provide here new data on this issue by showing that there was little difference between the impedance measured in intact (arborized) neurons or in (non-arborized) neurons with greatly simplified dendritic morphology. Therefore, the presence of dendrites is not a plausible cause to explain the deviations from resistivity.

The third conclusion, that the underlying mechanism is the ionic diffusion in Debye layers around the membrane, is mostly supported by the experiments in primary cell cultures where no intact neuropil is present. In these conditions, the medium is almost devoid of glial cells or neighboring neurons, and can be considered close to homogeneous saline. We also observed a frequency dependence in such conditions, when an intracellular recording was present, but not in saline with two electrodes placed in ACSF (see Section 3.2). Therefore, we find that the presence of a membrane induces a frequency dependence, which we attribute here as mainly due to the presence of Debye layers around the membrane. Debye layers not only constitute the basis of the membrane capacitance, but they also are characterized by ionic diffusion which participates to maintain the membrane potential. Our interpretation is that, when current flows from the intracellular electrode, the corresponding ions induce local concentration changes in Debye layers, which will re-equilibrate by ionic diffusion. This increases the modulus of the impedance and introduces a frequency dependence which signature can be seen as a diffusive (Warburg) impedance. However, it is important to note that this impedance is a linear approximation, and thus, cannot capture the ratio $V / I^{g}$ for large variations of the membrane potential (such as during spikes; see discussion in ref. [19]).

The fourth conclusion is that it was necessary to include capacitive effects to account for differences between the different preparations. These effects were not necessary for a cell in a homogeneous medium (in culture), but were required to fit acute brain slice conditions when the the electrodes were very close (about $10 \mu \mathrm{m}$ ). 
On the other hand, no additional capacitive effect was seen between the extracellular electrode and the ground. This suggests the possibility that both intracellular and extracellular media have a significant diffusive component. We could fit the measurements assuming a different cutoff frequency between the media (about $5 \mathrm{~Hz}$ intracellular, and $40 \mathrm{~Hz}$ extracellular). This difference suggests that the medium is more tortuous intracellularly compared to the extracellular medium.

It is important to note that the present results seem in agreement with the principle of least constraint of Gauss, according to which the introduction of constraints modifies the least as possible the movement of a system. In electromagnetism, the application of this principle means that the majority of charges will follow the path with the lowest impedance ${ }^{7}$. In the case of Logothetis et al. and Miceli et al. measurements $[5,6]$, the magnitude of the minimal extracellular impedance would be of the same order as ACSF, which is a similar situation as our measurement between the extracellular electrode and the ground. In the case of Gomes et al. [9], as in the present paper, the modulus of the minimal impedance between the intracellular and extracellular electrodes would be much larger because the charges are constrained to flow across the cell membrane, and not just flowing exclusively in the extracellular medium.

Taking together our results in different preparations, we conclude that, for the frequency range of electrophysiological phenomena (frequencies $\nu<10 \mathrm{kHz}$ ), there is a very significant frequency dependence of the electric parameters in intracellular and extracellular media. Our experiments show that this significant frequency dependence should be taken into account when current flows across a cell membrane. On the other hand, the experiments show that there is very little frequency dependence if the current does not flow across a membrane. This major difference suggests that impedance measurements should necessarily give different results if they are performed extracellularly, or using an intracellular recording. This could potentially reconcile contradictory measurements and also answer some of the questioning about the origin of the LFP signal [41].

This raises the question of which impedance is the most physiologically pertinent, the impedance of the medium alone, measured with extracellular electrodes, or the impedance between intracellular and extracellular media? If the point of interest is to relate neuronal activity (ionic currents) with the extracellular potentials, then the relevant impedance is the intracellular-extracellular impedance as measured here. Indeed, the ionic currents in the membrane have to flow through a complex environment (Debye layers, various obstacles), which are important to generate the extracellular potential (which will "sense" the current after its interaction with the complex environment). The impedance measured here correctly captures this effect. On the other hand, impedances measured with extracellular electrodes inform about how the extracellular medium reacts to currents injected extracellularly and does not reflect the situation with natural (membrane) currents. Thus, our study comple-

\footnotetext{
${ }^{7}$ For example, if we have two resistances in parallel, the majority of the charges will go through the smallest resistance. In electromagnetism, the principle of least constraint can be seen as a generalization of this example [32].
} 
ments previous extracellular measurements, and goes further in biophysical realism by measuring the impedance pertinent for the genesis of extracellular potentials.

\section{Author contributions}

CP and LV did the experiments, AD and CB conceptualized the study, CB did the analysis, and all authors contributed to interpret the analysis and to write the manuscript.

\section{Acknowledgements}

We thank Peter Vanhoutte, Nicolas Heck and Lisa Huet for help with the cell cultures. Research supported by the CNRS, INSERM, Collège de France, the European Community (grants H2020-785907 and H2020-945539) and the ANR (grant PARADOX).

\section{References}

[1] Buzsáki, G., Anastassiou, C., and Koch, C. 2012. The origin of extracellular fields and currents: EEG, ECoG, LFP and spikes. Nature Reviews Neurosci. 13, 407-420.

[2] Makarova, J., Gomez-Gala, M., and Herreras, O. (2008). Variations in tissue resistivity and in the extension of activated neuron domains shape the voltage signal during spreading depression in the CA1 in vivo. European Journal of Neuroscience 27: 444456.

[3] Rall, W.,G.M. Shepherd.1968. Theoretical Reconstruction of Field Potentials and Dendrodendritic Synaptic Interactions in Olfactory Bulb J Neurophysiol. Nov;31(6):884-915. doi: 10.1152/jn.1968.31.6.884.

[4] Ranck, J..1963. Analysis of specific impedance of rabbit cerebral cortex. Exp. Neurol. 7, 144-152.

[5] Logothetis, N.K.,C. Kayser, and A. Oeltermann. 2007. In vivo measurement of cortical impedance spectrum in monkeys: implications for signal propagation. Neuron 55: 809823.

[6] Miceli, S., T.V. Ness, G.T. Einevoll, and D. Schubert . 2017. Impedance Spectrum in Cortical Tissue: Implications for Propagation of LFP Signals on the Microscopic Level. eNeuro,Jan-Feb; 4(1).

[7] Gabriel, S., R.W. Lau, and C. Gabriel.1996. The dielectric properties of biological tissues: II. Measurements in the frequency range $10 \mathrm{~Hz}$ to $20 \mathrm{GHz}$. Phys. Med. Biol., 41, 2251 . 
[8] Wagner, T., U. Eden, J. Rushmore, C.J. Russo, L, Dipietro, F. Fregni, S. Simon, S. Rotman, N.B. Pitskel, C. Ramos-Estebanez, A. Pascual-Leone, A.J. Grodzinsky, M. Zahn, and A. Valero-Cabre. 2014. Impact of brain tissue filtering on neurostimulation fields: a modeling study. Neuroimage, Jan 15; 85(0 3): 1048-1057.

[9] Gomes, JM, C. Bédard, S. Valtcheva, MJ. Nelson, V. Khokhlova, P. Pouget, L. Venance, T. Bal and A. Destexhe. 2016. Intracellular impedance measurements reveal non-ohmic properties of the extracellular medium around neurons. Biophys. J. 110: 234-246.

[10] Bédard, C., H. Kröger, A. Destexhe. 2004. Modeling extracellular field potentials and the frequency-filtering properties of extracellular space. Biophys. J. 64:1829-1842.

[11] Bedard, C., H. Kroger and A. Destexhe. 2006. Model of low-pass filtering of local field potentials in brain tissue Phys. Rev. E 73:051911.

[12] Bédard, C., Destexhe, A.. 2009. Macroscopic models of local field potentials and the apparent $1 / f$ noise in brain activity. Biophys. J. 96(7):2589-4608.

[13] Bedard, C., S. Rodrigues, N. Roy, D. Contreras, A. Destexhe. 2010. Evidence for frequency-dependent extracellular impedance from the transfer function between extracellular and intracellular potentials: intracellular-LFP transfer function J. Comput. Neurosci. Dec;29(3):389-403.

[14] Dehghani, N., C. Bedard, S.S. Cash, E. Halgren, A. Destexhe . 2010. Comparative power spectral analysis of simultaneous elecroencephalographic and magnetoencephalographic recordings in humans suggests non-resistive extracellular media. J. Comput. neurosci. 29 (3): 405-421.

[15] Bedard, C., JM. Gomes, T. Bal, A. Destexhe . 2017. A framework to reconcile frequency scaling measurements, from intracellular recordings, local-field potentials, up to EEG and MEG signals. Journal of Integrative Neuroscience 16 (1): 3-18.

[16] Barbour, B. 2017. Analysis of Claims that the Brain Extracellular Impedance Is High and Non-resistive, Biophys. J. Volume 113, Issue 7, 1636-1638

[17] Nelson, MJ.,P. Pouget, E. A. Nilsen,C. D. Patten, and J. D. Schall. 2008. Review of Signal Distorsion through Metal Microelectrode Recordinf Circuits and Filters, J Neurosci Methods, Mar 30; 169(1): 141-157.

[18] Nelson, MJ, S. Valtcheva, L. Venance. 2017. Magnitude and behavior of cross-talk effects in multichannel electrophysiology experiments. J Neurophysiol. 118: 574-594.

[19] Bédard, C., A Destexhe . 2011. Generalized theory for current-source-density analysis in brain tissue, Phys. Rev. E 84 (4):041909.

[20] Le Bellac, M., Lévy-Leblond J.M. . 1973. Galilean electromagnetism. Nuovo Cim B $14,217-234$.

[21] Bédard, C. and Destexhe, A. . 2013. Generalized cable theory for neurons in complex and heterogeneous media. Physical Review E 88:022709. 
[22] Rall, W.. 1995. The theoretical foundations of dendritic function.MIT Press, Cambridge, MA.

[23] Tuckwell, H.C.. 1988. Introduction to Theoretical Neurobiology: Linear Cable Theory and Dendritic Structure. Cambridge University Press, Cambdridge, UK.

[24] Bartels, R.H., J.C. Beatty, B.A. Barsky. 1987. An Introduction to Splines for uses in Computer Graphics and Geometric Modeling. Ed. Morgan Kaufman Publishers, Inc, Los Altos, California, 485 p.

[25] Johnston, D. and Wu S.M. 1995. Foundations of Cellular Neurophysiology, Ed. MIT Press.

[26] Maxwell, J.C. . 1873 A Treatise on Electricity and Magnetism. Clarendon Press, Oxford. Vol. 1

[27] Priou, A. . 1992. Dielectric properties of heterogeneous materiels . Progress in Electromagnetics Research, Elsevier, New York.

[28] Vasiliev, A. M.. 1984. Introduction To Statistical Physics, Ed. Mir.

[29] Nicholson, C. . 2005. Factors governing diffusing molecular signals in brain extracellular space. J. Neural Transm. 112, 29-44 .

[30] Destexhe, A. and Bédard C.. 2013. Local Field Potential. Scholarpedia, http://www.scholarpedia.org/article/Local_field_potential

[31] Kalachnikov, S.. 1980. Electricity, Ed. Mir.

[32] Goldstein, H., C.P. Poole, J.L. Safko. 2001. Classical Mechanics (3rd Edition) . Ed. Addison Wesley, 647 p.

[33] Foster, K. R., H.P. Schwan . 1989. Dielectric properties of tissues and biological materials: A critical review. Critical Reviews in Biomedical Engineering 17(1):25-104.

[34] Bedard, C., Destexhe A. .2014. Mean-field formulation of Maxwell equations to model electrically inhomogeneous and isotropic media Equations to Model Electrically. Journal of Electromagnetic Analysis and Applications 6 (10), 296.

[35] Bedard, C., Destexhe A. .2014. Generalized cable formalism to calculate the magnetic field of single neurons and neuronal populations. Physical Review E 90 (4):042723.

[36] Oberhettinger, F. and L. Badii. 1973. Tables of Laplace Transforms. Springer-Verlag Berlin Heidelberg, New York.

[37] Rall, W. .1995. The Theoretical Foundation of Dendritic Function. Segev, I., J. Rinzel and G.M. Shepherd, ed. MIT Press, Cambridge MA.

[38] Hines, M.L., and N.T. Carnevale. 1997. The NEURON simulation environment. Neural Computation 9: 1179-1209. 
[39] Nelson, MJ., C. Bosch, L. Venance, and P. Pouget . 2013. Microscale Inhomogeneity of Brain Tissue Distorts Electrical Signal Propagation. The Journal of Neuroscience,33(7):2821-2827.

[40] Hrabetova S, Cognet L, Rusakov DA, Nagerl UV. 2018. Unveiling the extracellular space of the brain: from super-resolved microstructure to in vivo function. J Neurosci. 38: 9355-9363.

[41] Herreras, O. 2016, Local Field Potentials : Myths and Misunderstandings. Front. Neural Circuits, 10 : 101. https://doi.org/10.3389/fncir.2016.00101 


\section{Supplemental Information}

\section{Appendices}

\section{A Equivalent impedance between the extracellular electrode and the ground}

In this appendix, we give the explicit expressions to calculate the impedance between the extracellular electrode and the ground in the different experimental conditions considered.

When measuring the equivalent impedance, we have $\left(Z_{a}\left\|Z_{s}\right\| Z_{d}\right) \oplus Z_{g}$, where $Z_{g}$ is the impedance between the ground and the first isopotential surface that surrounds the neuron. $Z_{a}$ is the impedance of the extracellular medium in contact with the isopotential surface $S_{2}, Z_{s}$ is the impedance of the current flowing through the soma membrane in contact with surface $S_{2}$, and $Z_{d}$ is the input impedance of the dendritic tree relative to a reference outside the neuron(see Fig. 7).

Thus, we obtain

$$
\frac{V_{i}}{I_{s}^{g}}=Z_{e q}=\frac{Z_{a} Z_{s} Z_{d}}{Z_{a} Z_{s}+Z_{s} Z_{d}+Z_{d} Z_{a}}+Z_{g}
$$

where $I_{s}^{g}=I_{a}^{g}+I_{s}^{g}+I_{d}^{g}$. $I_{s}^{g}$ is the generalized current produced by the current source, because in our experiments, the generalized current conservation applies. Note that this does not account for charges created by chemical reactions [35]. We have

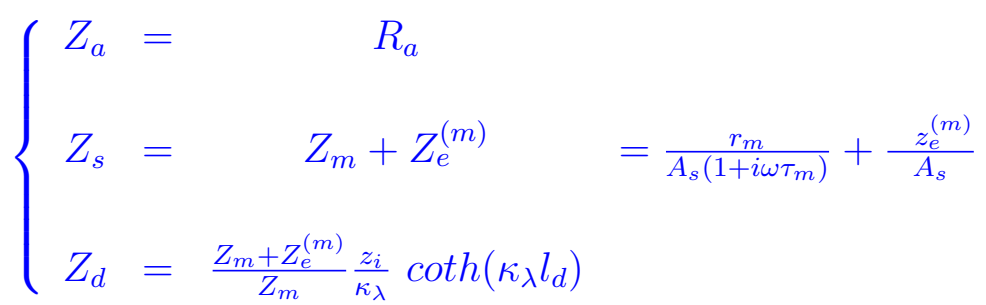

Here, we calculated $Z_{d}$ as follows. The part of the current source that flows through the dendrite before eventually going to the ground $\left(I_{d}^{g}\right)$ is such that we obtain $Z_{i n}^{d}=\frac{V_{m}}{I_{d}^{g}}=\frac{z_{i}}{\kappa_{\lambda}} \operatorname{coth}\left(\kappa_{\lambda} l_{d}\right)$ where $V_{m}$ is the somatic membrane potential at the basis of the dendrite [21]. In addition, applying the generalized current conservation gives the following equality:

$$
\frac{V_{i}^{S_{2}}}{Z_{m}+Z_{e}^{(m)}}=\frac{V_{m}}{Z_{m}}
$$

where the potential $V_{i}^{S_{2}}$ is taken at the isopotential surface $S_{2}$. Thus, we have approximately:

$$
Z_{d}=\frac{V_{i}}{I_{d}^{g}}=\frac{Z_{m}+Z_{e}^{(m)}}{Z_{m}} Z_{i n}^{d}=\frac{Z_{s}}{Z_{m}} Z_{i n}^{d}
$$




\section{B RC circuit in series with a resistance}

In this appendix, we compare the $\mathrm{RC}$ model $((R \| C))$ with the $\mathrm{RC}$ model in series with a resistance $((R \| C) \oplus R *)$. One can see from Fig. B.1 that the impedances of these two models are similar for small frequencies. However, they differ at high frequencies relative to the cut-off frequency of the RC circuit. It is important to also consider that the phase of the RC model tends to $-90^{\circ}$ when frequency tends to $\infty$, but it tends to $0^{\circ}$ for the $\mathrm{RC}$ model in series with a resistance.
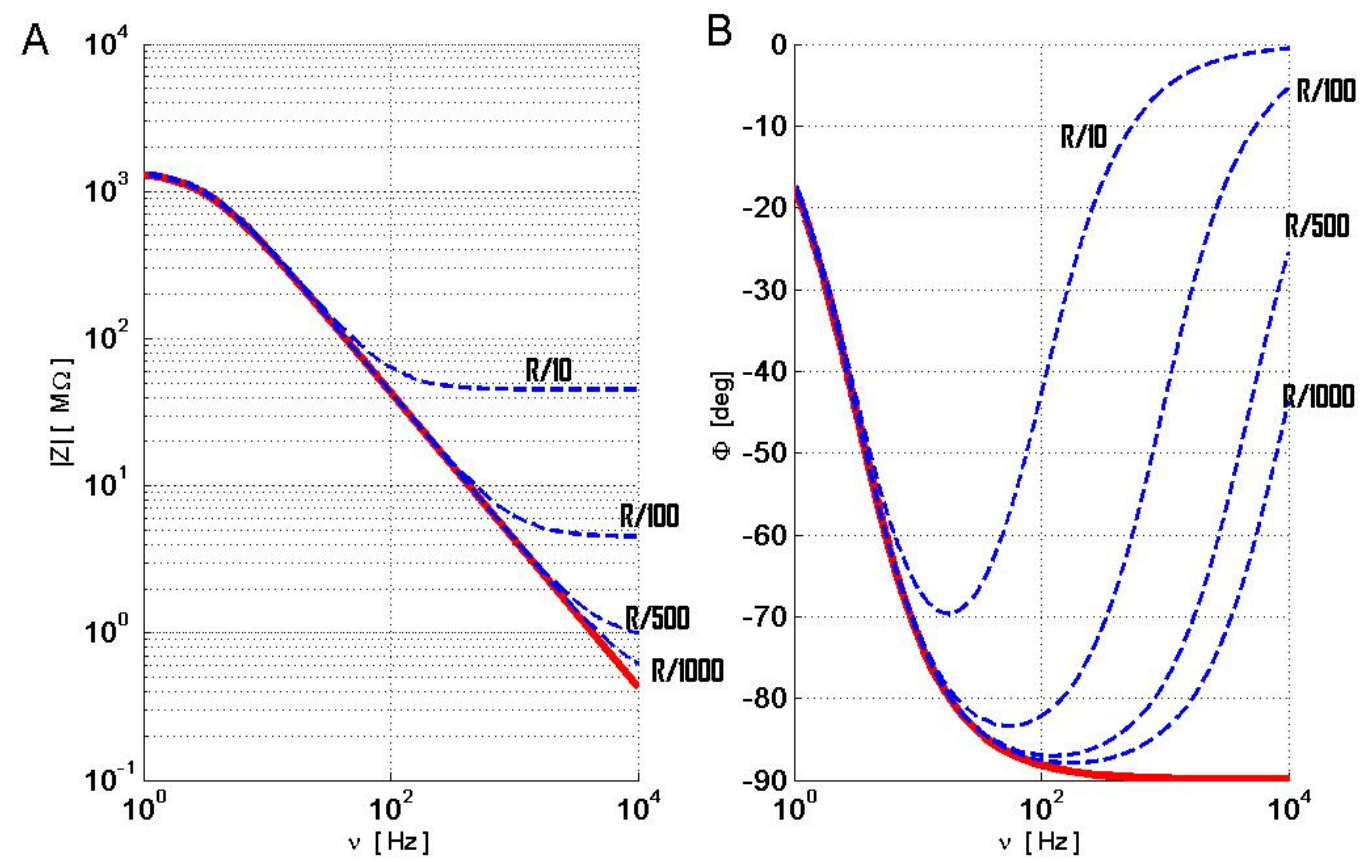

Figure B.1: Comparaison betwen the impedances of the model RC $\|$ (red) and of the model RC $\|$ in series with a resistance (blue). The modulus (A) and phase (B) are shown as a function of frequency.

\section{Diffusive impedance in heterogeneous media for a spherical source}

In this appendix, we present the theoretical expression of the macroscopic impedance in the case of a diffusive model [19].

In a previous publication [19], we have shown that the macroscopic diffusive impedance (also called Warburg impedance) is derived by a linear approximation of the ratio $\frac{V}{I^{g}}$, where $V$ is the potential difference between the two measurement points. This derivation took into account Boltzmann distribution and Ohm's law. The energy given to the charges divides into two parts: one dissipative part (calorific 
energy) and the part corresponding to the spatial arrangement and distribution of charges as a function of time. The first part is related to Ohm's law, and the second part to Nernst law.

The presence of a current source in a homogeneous medium breaks its homogeneity. Indeed, the charge distribution around the source cannot be considered constant. The application of Boltzmann's law in the quasistatic regime (in the sense of classical statistical thermodynamics), in the linear approximation, gives an impedance for a spherical current source of the form:

$$
Z_{\omega}=\frac{C k T}{\left(\frac{1}{R}+\sqrt{-i \frac{\omega}{<\beta>\left.\right|_{m}}}\right)}
$$

where $C$ is a constant which depends on the electric conductivity of the medium in the absence of the source, $R$ is the radius of the spherical source (which gives a curvature of $\left.1 / R^{2}\right), T$ is the absolute temperature in Kelvins, $\left.\langle\beta\rangle\right|_{m}$ is equivalent to an "effective" diffusion coefficient which is negative, and $k=1.38 \times 10^{-23} \mathrm{~J} /{ }^{\circ} \mathrm{K}$ is the Boltzmann constant (for more details, see [19]). This model is called "diffusive model", and is used here for the particular case of a spherical source.

By setting

$$
A_{w}=C R T
$$

and

$$
\omega_{w T}=-\frac{<\beta>\left.\right|_{m}}{R^{2}}
$$

we can write expression C.1 as above:

$$
Z_{w}=\frac{A_{w}}{1+\sqrt{i \frac{\omega}{\omega_{w T}}}}
$$

At constant temperature, the measurement of the impedance allows one to determine the values of $A_{w}$ and $\omega_{w T}=2 \pi \nu_{w T}$. The parameters $A_{w}$ and $\omega_{w T}$ are real.

However, we assumed

$$
Z_{w}=\frac{A_{w}}{1+\sqrt{i \frac{\omega}{\omega_{w T}}}}+R_{a s y m p}
$$

because the original derivation of the expression of the Warburg impedance in mean-field [19] considered the particular solution of the differential equations in mean-field, also called the "forced solution". The general solution is the sum of this particular solution and the solution of the homogeneous equation $\left(\nabla^{2} V_{\omega}=0\right)$. To take this into account, one needs to add a resistance in series with the forced solution $\left(R_{\text {asymp. }}\right)$. This asymptotic resistance appears at very large frequencies in the experimental measurements (see Results). 
A

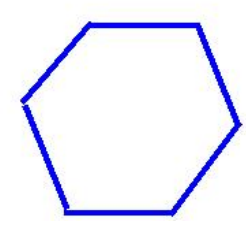

B

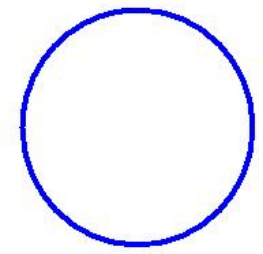

Figure D.1: Examples of how the curvature of isopotential surfaces determines the cutoff frequency of the impedance. A. Volume delimited by a plane (infinite curvature radius), resulting in a cutoff frequency near zero. B. Similar volume delimited by a border of constant curvature. In this case, the cutoff frequency is larger because it is inversely proportional to the curvature radius (see Appendix D ).

\section{Threshold frequency and surface curvature of the diffusive model in the general case}

In this appendix, we give some details about the relation between the threshold frequency in the diffusive impedance (expression C.5) and the curvature at a given point of a surface $S$. In other words, we show how to apply the diffusive model to surfaces that are non-spherical.

The diffusive model of Appendix C can be applied to an arbitrary surface because we can build an approximately continuous surface $\mathcal{S}$ by the sum of portions of spherical surfaces centered on different points of $\mathcal{S}$, where the curvature corresponds to that of $\mathcal{S}$. Note that the smaller the intrinsic curvature of a surface, the smaller is the threshold frequency of that surface ${ }^{8}$.

For example, if we have a surface $\mathcal{S}$ composed of two spherical portions $\left(\mathcal{S}_{1}\right.$ and $\left.\mathcal{S}_{2}\right)$ of very different radius, the diffusive impedance diffusive as sensed by the surface is equal to the two impedances of each portion in parallel, because the current divides between both of them. It follows that $Z_{S}=Z_{S_{1}} \| Z_{S_{2}}$, with:

$$
Z_{S}=\frac{A_{w}}{1+\sqrt{i \frac{\omega}{\omega_{w T}}}}
$$

where

$$
\left\{\begin{array}{c}
A_{w}=\frac{A_{w_{1}} A_{w_{2}}}{A_{w_{1}}+A_{w_{2}}} \\
\frac{1}{\sqrt{\omega_{w T}}}=\frac{A_{w_{1}}}{\frac{\sqrt{\omega_{2}}}{\omega_{w_{2}}}+\frac{A_{w_{2}}}{\sqrt{\omega_{s_{1}}}}}
\end{array}\right.
$$

If the surfaces $\mathcal{S}_{1}$ and $\mathcal{S}_{2}$ have the same impedance, then the impedance of $\mathcal{S}$ is twice smaller, but the threshold frequency remains the same. If each surface displays a similar Warburg amplitude but with different threshold frequencies, then we obtain

\footnotetext{
${ }^{8} \mathrm{~A}$ similar approach is classically used to model the electrical point effect [31].
} 


$$
\frac{1}{\sqrt{\omega_{w T}}}=\frac{1}{\sqrt{\omega_{s_{1}}}}+\frac{1}{\sqrt{\omega_{s_{2}}}}
$$

It follows that if we approximate a given surface with a set of $N$ spherical portions of same Warburg amplitude, the threshold frequency is given by:

$$
\frac{1}{\sqrt{\omega_{w T}}}=\sum_{i=1}^{N} \frac{1}{\sqrt{\omega_{s_{i}}}}
$$

Thus, the portions of surface with the smallest curvature will determine the threshold frequency of the ensemble, Consequently, it is possible to obtain a very small threshold frequency, even in a domain of a very small volume (see Fig. D.1).

\section{E Macroscopic impedance relative to ground}

In this appendix, we consider the macroscopic impedance as sensed by the electrode injecting the current in the soma, via the dendrite, before reaching the ground, $Z_{\text {in den }}^{\text {ground }}$, and the impedance as seen by the current going to the ground independently of the dendrite, $Z_{\text {out den }}^{\text {ground }}$, for a ball-and-stick model in a resistive extracellular medium. Note that the impedance between the soma and the ground is given by $Z_{\text {in den }}^{\text {ground }} \| Z_{\text {out den }}^{\text {ground }}$

We also consider that the cytoplasm is resistive, as well as the Debye layers surrounding the membrane. We will consider the experimental measurements of Section 3.2. Importantly, in the present experiments, the impedance between the cell and the ground should be calculated in an "open" configuration, because the current injected in the neuron flows to the ground without looping back to the neuron.

We numerically compared the impact of the two configurations, open and closed, at the basis of the dendrite (stick). In particular, the parameter $\kappa_{\lambda}=\kappa / \lambda$ is a good indicator to evaluate the differences between the two configurations.

For this purpose, we first assumed that the extracellular impedance $z_{e}^{(m)}$ has the same value at every point in the membrane in soma and dendrites. This hypothesis is reasonable if the neuron is physically smaller than the geometrical dimensions of the experimental preparation. Indeed, this parameter measures the impedance of the extracellular medium as sensed by the membrane, as defined by $z_{e}^{(m)} / d S$ which is the impedance between $d S$, a differential element of membrane, and the ground. 

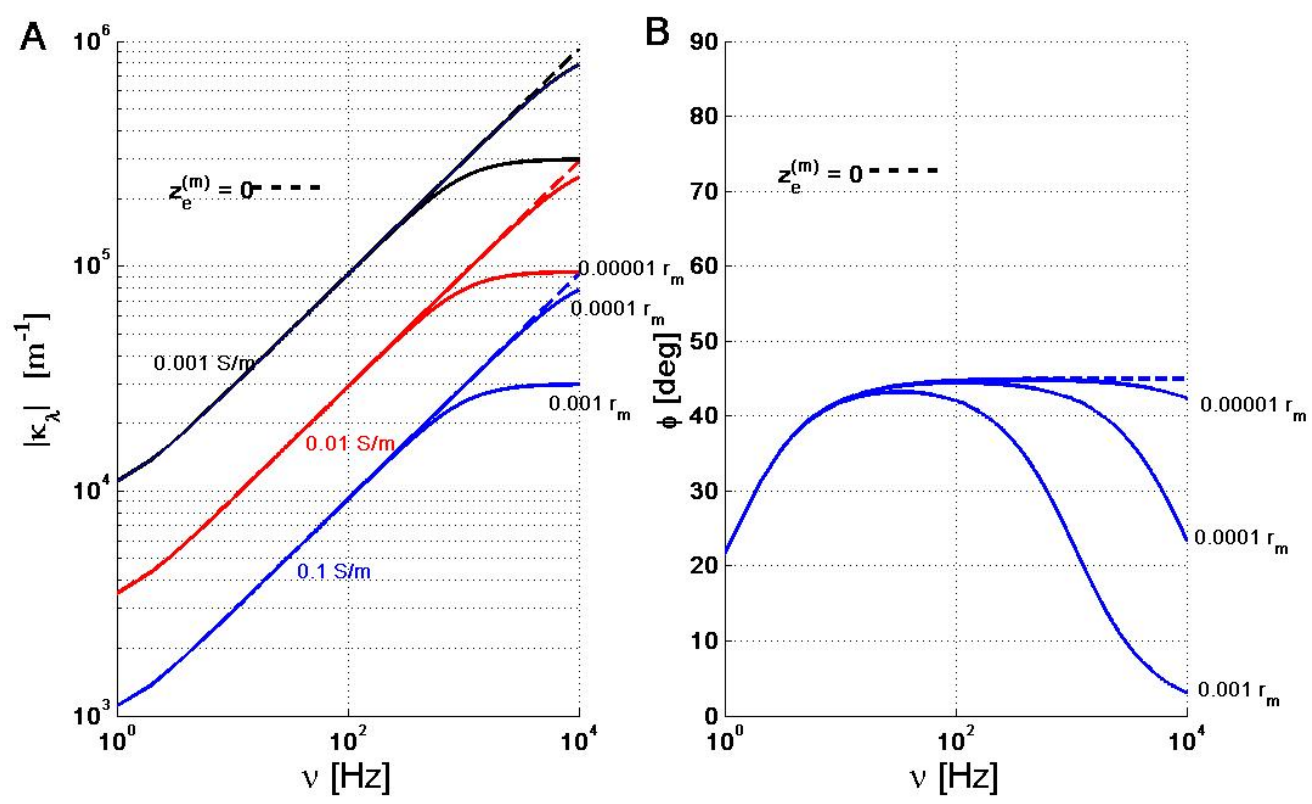

Figure E.1: Graph of $\frac{\kappa}{\lambda}$ as a function of frequency for the ball-and-stick model. Here, $\kappa=\sqrt{1+i \omega \tau_{m}}$, the soma has a radius of $10 \mu \mathrm{m}$, while the length and diameter of the stick are respectively of $600 \mu \mathrm{m}$ and $3 \mu \mathrm{m}$. In this example, the membrane time constant $\tau_{m}$ is of $30 \mathrm{~ms}, c_{m}=0.01 \mathrm{~F} / \mathrm{m}^{2}$ is the specific membrane capacitance, and $z_{e}^{(m)}=k r_{m}$ with $k=0.001,0.0001,0.00001$. The dashed lines correspond to the open configuration, and continuous lines to the closed configuration. The electric conductivity of the cytoplasm $\sigma_{e}^{n m}$ corresponds to the different colors, Blue: $\sigma_{e}^{n m}=$ $0.1 \mathrm{~S} / \mathrm{m}$, Red: $\sigma_{e}^{n m}=0.01 \mathrm{~S} / \mathrm{m}$, Black: $\sigma_{e}^{n m}=0.001 \mathrm{~S} / \mathrm{m}$. Note that in the case $z_{e}^{(m)}=0.00001 r_{m}$ is approximately equivalent to a supraconductive medium, for frequencies smaller than $10 \mathrm{kHz}$.

According to the generalized cable theory [21], for a resistive medium, we have:

$$
\left\{\begin{array}{l}
r_{m}=\frac{e_{m}}{\sigma_{e}^{(m n)}} \\
z_{e}^{(m)}=\frac{e_{c}}{\sigma_{e}^{(c)}}
\end{array}\right.
$$

where $e_{m}$ and $e_{c}$ are the thickness of the membrane and of Debye layers, respectively. $\sigma_{e}^{(m n)}$ and $\sigma_{e}^{(c)}$ are the mean electric conductivity of the membrane and of the extracellular medium (comprising Debye layers), respectively. Debye layers have a high density of ions, and thus have a different conductivity than the "bulk" of the medium. The ions around the membrane are distributed according to Boltzmann distribution, forming Debye layers, and the diffusive model must be taken into account in this case (Appendices $\mathrm{C}$ and D). The electric conductivity is much lower in Debye layers compared to the other parts of the extracellular medium, which is considered homogeneous away of Debye layers. We assumed a thickness equivalent to that of Debye layers in the expression of $z_{e}^{(m)}$ (see Eq. E.1).

However, in this appendix, we neglect the possible frequency dependence and model the impedance of Debye layers with a resistance, as if the threshold frequency was 
very large. The goal here is to determine, as simple as possible, the physical consequences of the magnitude of $\left|z_{e}^{(m)}\right|$ relative to $r_{m}$ on the current division between the soma and the dendritic stick.

According to expressions E.1, we obtain:

$$
z_{e}^{(m)}=\frac{e_{c}}{e_{m}} \frac{\sigma_{e}^{(m n)}}{\sigma_{e}^{(c)}} r_{m}
$$

where $r_{m}=\tau_{m} / c_{m}=100 \tau_{m}$ (with $\left.c_{m}=0.01 \mathrm{~F} / \mathrm{m}^{2}\right)$.

For a value of $\tau_{m}=30 \mathrm{~ms}, e_{c}=0.1 e_{m}$ and $\sigma_{e}^{(m n)}=10^{-2} \sigma_{e}^{(c)}$, we obtain $z_{e}^{(m)}=$ $0.003 \Omega \mathrm{m}^{2}$. This value gives the order of magnitude of the physical effects on the impedances $Z_{\text {in den }}^{\text {ground }}$ and $Z_{\text {out den }}^{\text {ground }}$. The value of the membrane time constant is that of the experiments presented here.

Next, from the evolution of the electric conductivity of the cytoplasm, we consider three different values: $0.1,0.01$ and $0.001 \mathrm{~S} / \mathrm{m}$ (see Fig. E.2 ) The first value approximately corresponds to that of ACSF for a temperature of $37^{\circ} \mathrm{C}$. The two other values are smaller, to simulate the fact that the cytoplasm is a heterogeneous medium (presence of organites), which creates a tortuosity, as well as electric polarization. These effects have been reported to diminish electric conductivity [26, 27].
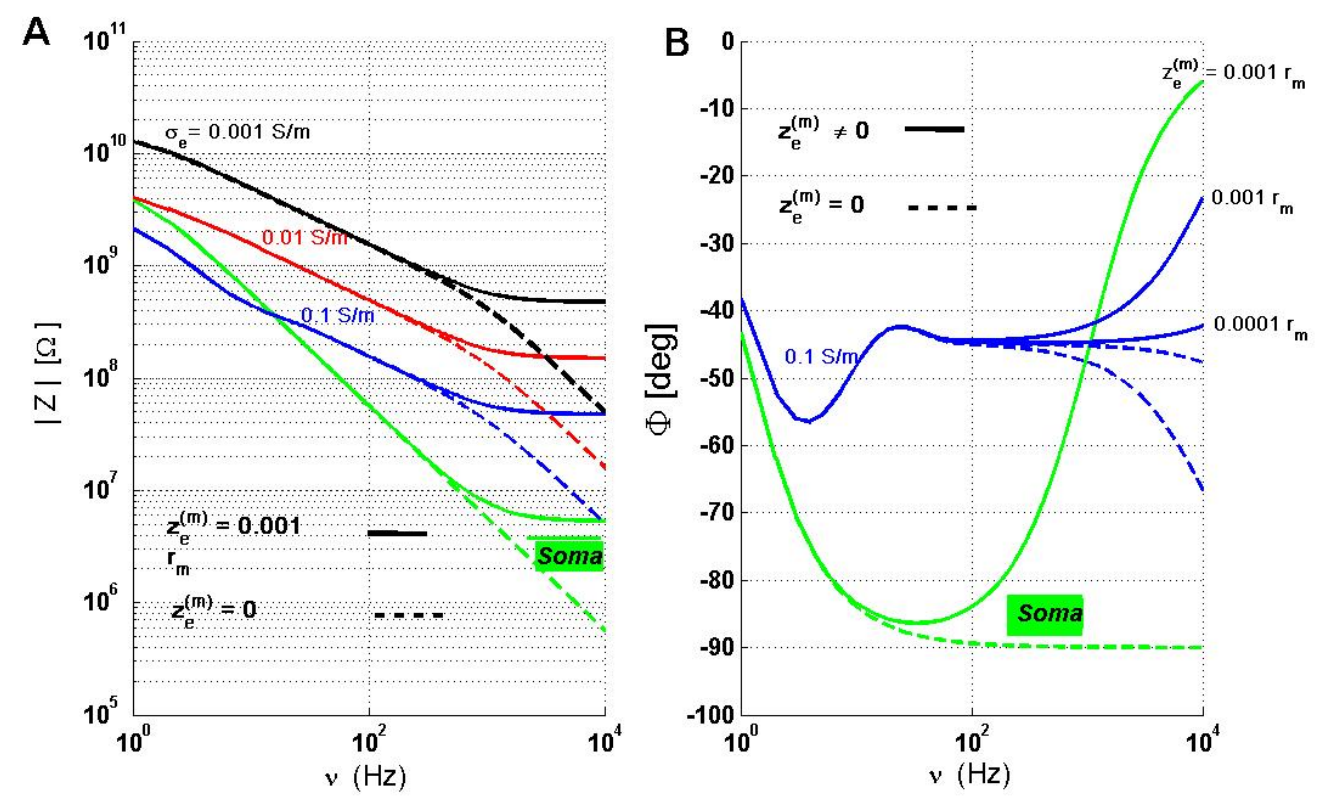

Figure E.2: Example of input impedance $Z_{\text {in den }}^{\text {ground }}$ and of soma impedance $Z_{\text {soma }}^{\text {ground }}$. Here, $z_{e}^{(m)}=k r_{m}$ and $k=0.001,0.0001$. The electric conductivity of the cytoplasm is $\sigma_{e}^{n m}=0.1,0.01,0.001 \mathrm{~S} / \mathrm{m}$. The other parameters are the same as in Fig. D.1. The dotted lines correspond to the case $z_{e}^{(m)}=0$, which is equivalent to neglect Debye layers; solid lines correspond to a resistive model, with Debye layers taken into account.

Figure E.2 shows examples of the input impedance in the following conditions. 1) The open and closed configurations give very different results when $z_{e}^{(m)}>$ $0.01 \tau_{m} \Omega m^{2}$, otherwise the differences are small for parameters $\kappa_{\lambda}$. Note that the 
case $z_{e}^{(m)}<0.00001 r_{m}$ is as if Debye layers were inexistent for frequencies smaller than $10 \mathrm{kHz}$. 2). For $\sigma_{e}^{(\mathrm{nm})}=0.1 \mathrm{~S} / \mathrm{m},\left|Z_{\text {in den }}^{\text {ground }}\right|>\left|Z_{\text {soma }}^{\text {ground }}\right|$ if $\nu>100 \mathrm{~Hz}$, for $\sigma_{e}^{(n m)}=0.01 \mathrm{~S} / \mathrm{m}$. This inequality holds up to about $1 \mathrm{~Hz}$. For $\sigma_{e}^{(n m)}=0.001 \mathrm{~S} / \mathrm{m}$, the modulus of the dendrite (stick) impedance is much larger than that of the soma.

\section{F Apparent electric conductivity and permittivity}

In this appendix, we define the apparent electric conductivity and permittivity. In general, we have the following linking relations between the diffusion $\vec{j}^{f}, \vec{E}$ and the fields $\vec{D}, \vec{E}$ :

$$
\left\{\begin{array}{l}
\vec{j}^{f}(t)=\int_{-\infty}^{t} f_{\sigma}\left(t-t^{\prime}\right) \vec{E}\left(t^{\prime}\right) d t^{\prime} \\
\vec{D}(t)=\int_{-\infty}^{t} g_{\varepsilon}\left(t-t^{\prime}\right) \vec{E}\left(t^{\prime}\right) d t^{\prime}
\end{array}\right.
$$

This is obtained in the framework of a mean-field theory of Maxwell equations when the extracellular and intracellular media are linear and homogeneous [19, 34]. Note that the functions $f_{\sigma}$ and $g_{\varepsilon}$ are real functions which can model different physical phenomena, such as ionic diffusion, electric polarization, calorific (resistive) dissipation, etc. The integral expresses the fact that the free-charge current density and displacement current density at a given time $t$ are not only determined by the electric field at time $t$ but also by the whole history of its time variations. These functions are the inverse Fourier transform of electric conductivity and permittivity expressed in Fourier frequency space ${ }^{9}$. For example, for an ideal electric resistance, we have $f_{\sigma}(t)=\sigma_{e} \delta(t)$, for an ideal capacitance we have $g_{\varepsilon}(t)=\varepsilon_{s} \delta(t)$ (where $\sigma_{e}$ are $\varepsilon_{s}$ are constant in time). These two parameters are respectively the electric conductivity and permittivity. In these two ideal cases, the relations F.1 give the following equalities: $\vec{j}^{f}=\sigma_{e} \vec{E}$ and $D=\varepsilon_{s} \vec{E}$, where $\sigma_{e}$ and $\varepsilon_{s}$ are time independent. Note that these two ideal elements have no memory of the past (which is expressed by the Dirac deltas), and this is not generally the case of frequency-dependent electric conductivity and permittivity.

Consequently, we have in general, in Fourier frequency space:

$$
\left\{\begin{array}{l}
\vec{j}^{f}(\omega)=\sigma(\omega) \vec{E}(\omega) \\
\vec{D}(\omega)=\varepsilon(\omega) \vec{E}(\omega)
\end{array}\right.
$$

where $\sigma(\omega)$ and $\varepsilon(\omega)$ are respectively the Fourier transforms of $f_{\sigma}(t)$ and $g_{\varepsilon}(t)$. Because $f_{\sigma}(t)$ and $g_{\varepsilon}(t)$ are real functions, this implies in general $\sigma(-\omega)=\sigma^{*}(\omega)$ and $\varepsilon(-\omega)=\varepsilon^{*}(\omega)$. Here, the real parts are necessarily even functions and the imaginary parts are odd functions. The relations F.2 imply that the generalized

\footnotetext{
${ }^{9}$ Representing the electric parameters in Fourier frequency space is particularly efficient when the medium is linear because in this case the density of free-charge current and displacement current are proportional to $e^{i \omega t}$ if the electric field is also proportional to this term.
} 
current density is related to the electric field by:

$$
\vec{j}^{g}(\omega)=\vec{j}^{f}(\omega)+\vec{j}^{d}(\omega)=[\sigma(\omega)+i \omega \varepsilon(\omega)] \vec{E}(\omega)=\gamma(\omega) \vec{E}(\omega)
$$

where $\sigma=\sigma^{\prime}+i \sigma^{\prime \prime}$ and $\varepsilon=\varepsilon^{\prime}+i \varepsilon^{\prime \prime}$ are complex functions in general, while $\sigma^{\prime}, \sigma^{\prime \prime}$, $\varepsilon^{\prime}, \varepsilon^{\prime \prime}$ are real functions. We have the following particular cases: an ideal resistance is such that we have $\sigma(\omega)=\sigma_{e}$ (Ohm's law), an ideal capacitance corresponds to $\varepsilon(\omega)=\varepsilon$, so that $\sigma$ and $\varepsilon$ are real numbers and do not depend on frequency.

From these relations, we obtain:

$$
\gamma=\left(\sigma^{\prime}-\omega \varepsilon^{\prime \prime}\right)+i \omega\left(\varepsilon^{\prime}+\frac{\sigma^{\prime \prime}}{\omega}\right)=\sigma_{\mathcal{A}}+i \omega \varepsilon_{\mathcal{A}}
$$

which defines the apparent electric conductivity $\sigma_{\mathcal{A}}$ and the apparent electric permittivity $\varepsilon_{\mathcal{A}}$. In general, the apparent electric permittivity can be viewed as a type of resistance which depends on frequency and allows to calculate the dissipated power at a given frequency. The ratio $\varepsilon_{\mathcal{A}} / \sigma_{\mathcal{A}}$ can be used to evaluate the relaxation time of the medium. Note that this definition corresponds to the electric parameters measured in previous studies [7, 33]. In these experimental studies, the measurements are characterized by parameters $\sigma_{\mathcal{A}}$ and $\varepsilon_{\mathcal{A}}$ because we interpret the experimental measurements in a very heterogeneous medium as if it was a non-ideal resistance (which depends on frequency) in parallel with a non-ideal capacitance (which also depends on frequency). A heterogeneous medium can be modeled as a homogeneous medium where the parameters depend on frequency with respect to macroscopic measurements. This is analogous to classical thermodynamics where pressure and temperature can be used to characterize a physical system.

Note that the apparent electric permittivity tends to infinity if the imaginary part of the electric conductivity does not tend to zero at null frequency. This is not the case for an ideal resistance because the imaginary part of its electric conductivity is zero. However, for a diffusive (planar) impedance (with zero curvature, see Appendix D) the imaginary part of electric conductivity is non-zero, since in this case $\gamma=k \sqrt{\omega}(1+$ i) where $k$ is a constant.

By definition, the complex admittance $Y$ between the two arms of a plane capacitor with a given medium in between, is given by $\frac{A}{d}\left[\sigma_{\mathcal{A}}+i \omega \varepsilon_{\mathcal{A}}\right] . A$ is the arms area and $d$ is the distance separating them. If we assume that these geometrical dimensions do not generate boundary effects, the electric field between the arms is of $V / d$ where $V$ is the voltage difference between the arms of the capacitor. 

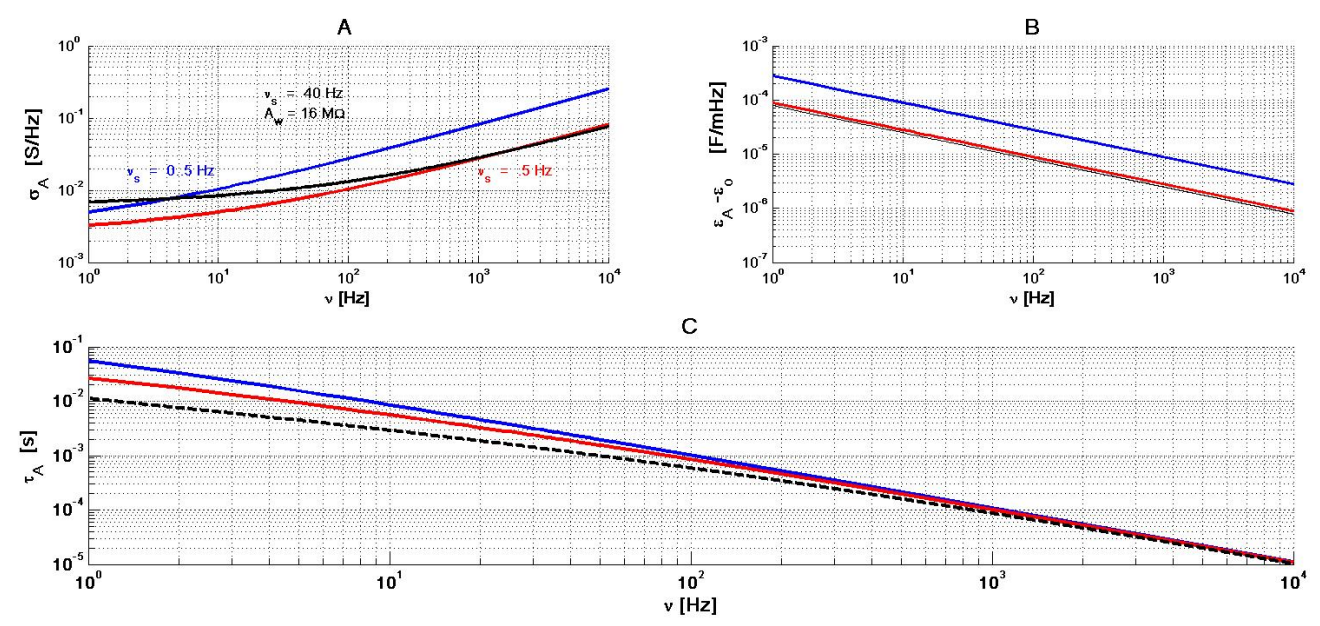

Figure F.1: Apparent conductivity and permittivity of a diffusive impedance. The red and blue curves correspond to $\nu_{w T}=.5 \mathrm{~Hz}$ and black curves correspond to $\nu_{w T}=40 \mathrm{~Hz}$. We have $A_{w}=16 \mathrm{M} \Omega$ and $A / d=10 \mu \mathrm{m}$ for all curves,

For example, the measurement of the apparent parameters of a medium with a diffusive impedance gives the following equality:

$$
Y=\frac{A}{d}\left[\sigma_{\mathcal{A}}+i \omega \varepsilon_{\mathcal{A}}\right]=\frac{1+\sqrt{i \frac{\omega}{\omega_{w T}}}}{A_{w}}
$$

It follows that the frequency dependence of the parameters is given by the following expressions:

$$
\left\{\begin{aligned}
k \sigma_{\mathcal{A}} & =1+\sqrt{\frac{\omega}{2 \omega_{w T}}} \\
k \varepsilon_{\mathcal{A}} & =\frac{1}{\sqrt{2 \omega \omega_{w T}}} \\
\tau_{\mathcal{A}} & =\frac{\varepsilon_{\mathcal{A}}}{\sigma_{\mathcal{A}}}=\frac{1}{\omega+\sqrt{2 \omega \omega_{w T}}}
\end{aligned}\right.
$$

where the constant $k$ is equal to $\frac{A A_{w}}{d}$.

Thus, the apparent electric conductivity tends to $k$, the electric permittivity tends to infinite, and the dielectric relaxation time tends to infinity for $\omega \rightarrow 0$. We conclude that if ionic diffusion is not negligible, then the linear approximation of its effect on the measured impedance is as if the dielectric relaxation time tends to infinity at null frequency (see Fig. F.1).

\section{G Ensemble of the measurements}

In this appendix, we show the ensemble of experimental results obtained in the different preparations. Figures G.1 (non-arborized neurons in culture), G.3 (arborized neurons in culture) and G.5 (arborized neurons in brain slices) respectively 
show the impedance of Region 1-2 (between the intracellular and extracellular electrodes). The same preparations are respectively shown in Figs. G.2, G.4 and G.6 for the modulus of the impedance of Region 2-3 (between the extracellular electrode and the ground). The values of the experimental parameters for the different experimental preparations are shown respectively in Table G.1, G.3 and G.5, while the values of the corresponding models are shown in Table G.2, G.4 and G.6.
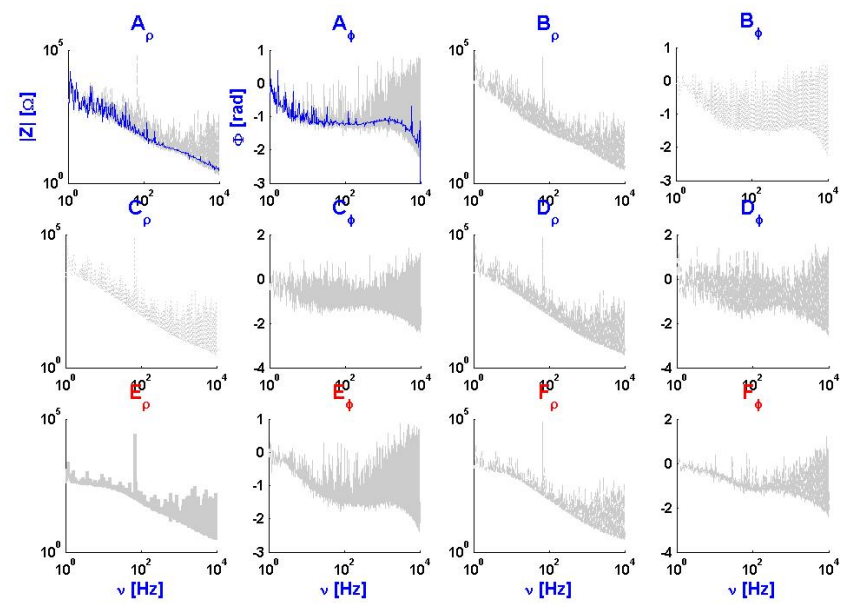

Figure G.1: Experimental measurement between intracellular and extracellular electrodes for 6 non-arborized neurons in culture. On the basis of the fits, two groups can be distinguished, one with $\tau_{m}$ around $30 \mathrm{~ms}$ and another group with 5-15 ms (see Tables G.1 and G.2). The blue curves are cubic spline fits of the experimental data.
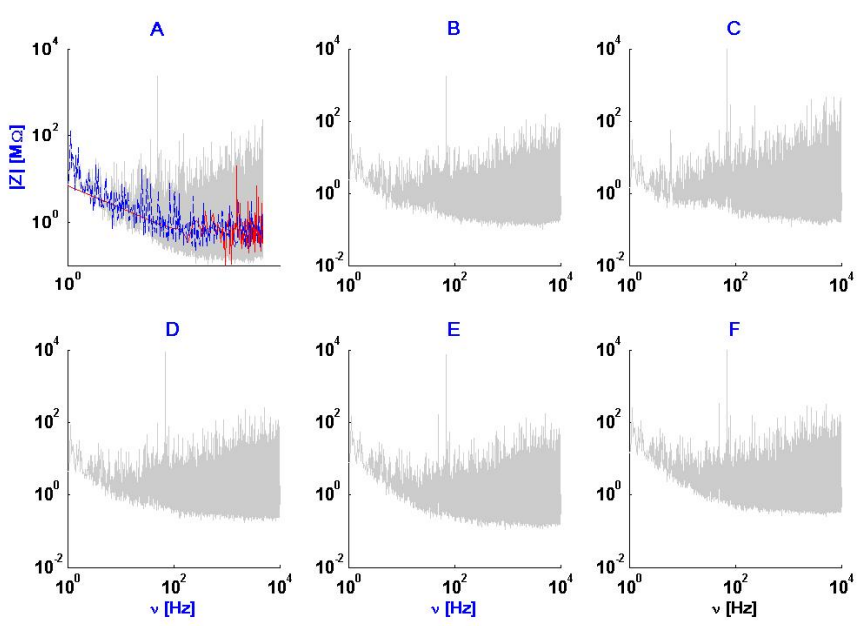

Figure G.2: Experimental measurements between extracellular and ground for 6 non-arborized neurons in primary cell culture, as shown in Tables G.1 and G.2. The blue curves are cubic spline fits to the logarithm of the experimental data, while the red curves are the direct cubic spline fits of the data. 


\begin{tabular}{|l|c|c|c|c|}
\hline & $\begin{array}{c}V_{m}(\text { mean } \pm \\
\text { standard deviation) } \\
\text { during white } \\
\text { noise injection }(\mathrm{mV})\end{array}$ & $\begin{array}{c}\sigma \text { of white } \\
\text { noise } \\
\text { current } \\
(\mathrm{pA})\end{array}$ & $\begin{array}{c}\text { Number } \\
\text { of sweeps }\end{array}$ & $\begin{array}{c}\text { Membrane } \\
\text { resistance }(\mathrm{M} \Omega) \\
\text { Membrane time } \\
\text { constant }(\mathrm{ms})\end{array}$ \\
\hline $\mathbf{A}: 2020021702$ & $-68.9 \pm 2.5$ & 10 & 18 & $\begin{array}{c}\text { NaN } \\
\text { NaN }\end{array}$ \\
\hline B $: 2020021801$ & $-64.6 \pm 6.4$ & 5 & 13 & $\begin{array}{c}2995 \\
117\end{array}$ \\
\hline C : 2020021901 & $-62.3 \pm 2.5$ & 10 & 20 & 900 \\
62
\end{tabular}

Table G.1: Individual experimental parameters for 6 non-arborized neurons in culture, shown in Fig. G.1. In the absence of measurements, a NaN is indicated.

\begin{tabular}{|c|c|c|c|c|c|c|c|}
\hline & $\begin{array}{c}R_{m} \\
{[M \Omega]}\end{array}$ & $\begin{array}{c}\tau_{m} \\
{[m s]}\end{array}$ & $\begin{array}{c}A_{w} \\
{[M \Omega]}\end{array}$ & $\begin{array}{c}\nu_{w T} \\
{[m s]}\end{array}$ & $\begin{array}{c}R_{\text {asymp }} \\
{[M \Omega]}\end{array}$ & $\begin{array}{c}\sigma_{d}^{\exp } \\
{[M \Omega]}\end{array}$ & $\begin{array}{c}\sigma_{r}^{\exp } \\
{[M \Omega]}\end{array}$ \\
\hline \hline $\mathbf{A}$ & 1000 & 30 & 625 & 0.1 & 4 & 3.4 & 142.9 \\
\hline $\mathbf{B}$ & 3000 & 30 & 2000 & 0.2 & 4 & 16 & 225.0 \\
\hline $\mathbf{C}$ & 3000 & 35 & 2000 & 0.05 & 6 & 3.5 & 210.0 \\
\hline $\mathbf{D}$ & 3000 & 25 & 2000 & 0.05 & 3 & 3.8 & 215.0 \\
\hline $\mathbf{E}$ & 3000 & 5 & 2000 & 0.7 & 2 & 2.9 & 58.3 \\
\hline $\mathbf{F}$ & 1000 & 15 & 670 & 0,1 & 2 & 2.2 & 179.0 \\
\hline
\end{tabular}

Table G.2: Parameters for the diffusive model for each non-arborized neuron in Table G.1. $\sigma_{r}^{\exp }$ and $\sigma_{d}^{\exp }$ are respectively the mean square error of resistive and diffusive models relative to the experimental measurements for each neuron . 

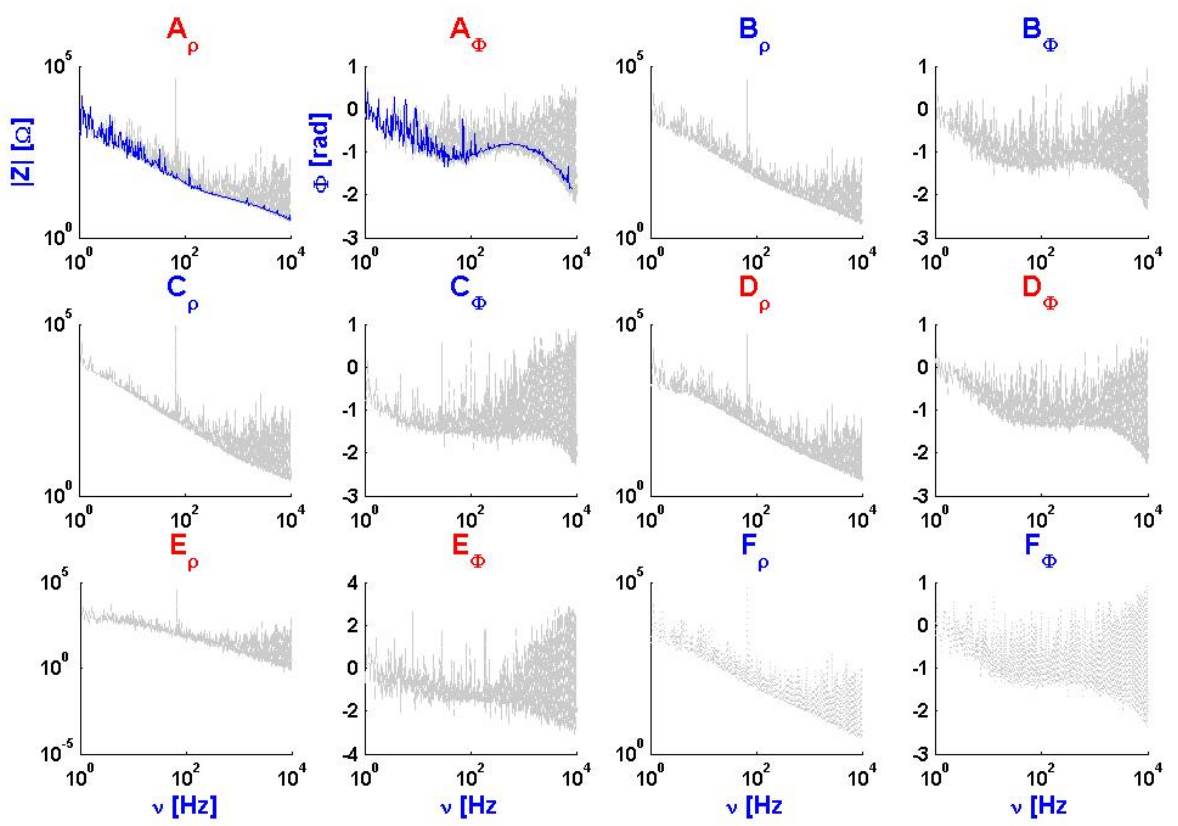

Figure G.3: Experimental measurements between intracellular and extracellular electrodes for 6 arborized neurons in primary cell culture (quasi-homogeneous medium). The blue curves are cubic spline fits of the experimental data.
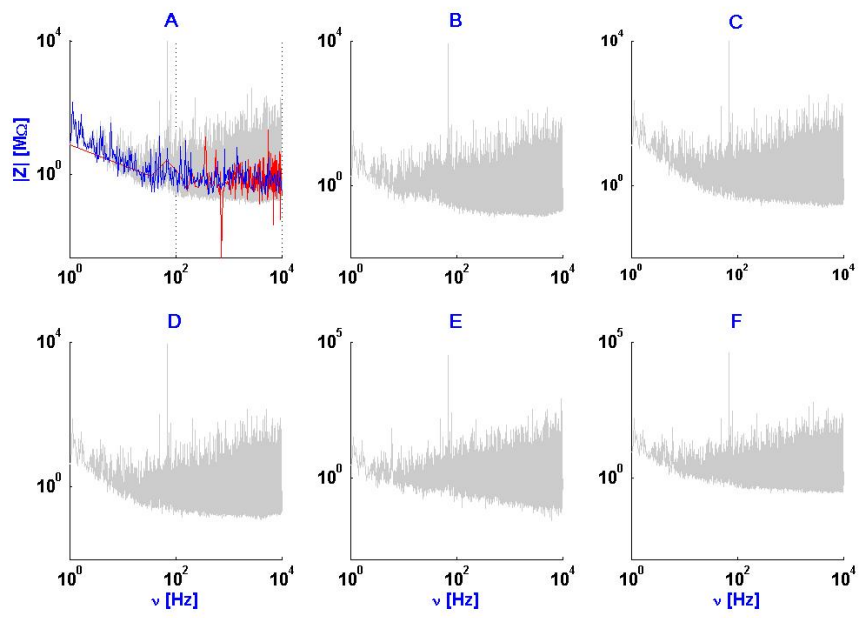

Figure G.4: Experimental measurements between extra and ground for 6 arborized neurons in culture, as shown in Tables G.3 and G.4. The blue curves are cubic spline fits to the logarithm of the experimental data, while the red curves are the direct cubic spline fits of the data. 


\begin{tabular}{|c|c|c|c|c|}
\hline & $\begin{array}{c}V_{m}(\text { mean } \pm \\
\text { standard deviation }) \\
\text { during white } \\
\text { noise injection }(\mathrm{mV})\end{array}$ & $\begin{array}{c}\sigma \text { of white } \\
\text { noise } \\
\text { current } \\
(\mathrm{pA})\end{array}$ & $\begin{array}{l}\text { Number } \\
\text { of sweeps }\end{array}$ & $\begin{array}{l}\text { Membrane } \\
\text { resistance }(\mathrm{M} \Omega) \\
\text { Membrane time } \\
\text { constant }(\mathrm{ms})\end{array}$ \\
\hline A : 2020021701 & $-66.0 \pm 2.6$ & 10 & 46 & $\begin{array}{c}549 \\
25\end{array}$ \\
\hline B : 2020021902 & $-68.1 \pm 4.0$ & 10 & 31 & $\begin{array}{c}1820 \\
67\end{array}$ \\
\hline C : 2020031501 & $-76.1 \pm 4.4$ & 5 & 28 & $\begin{array}{c}4737 \\
109\end{array}$ \\
\hline $\mathrm{D}: 2020031502$ & $-67.5 \pm 4.4$ & 10 & 60 & $\begin{array}{c}1347 \\
31\end{array}$ \\
\hline E : 2020031602 & $-66.7 \pm 2.1$ & 10 & 3 & $\begin{array}{c}793 \\
22\end{array}$ \\
\hline $\mathbf{F}: 2020031603$ & $-69.4 \pm 2.7$ & 5 & 26 & $\begin{array}{c}2220 \\
89\end{array}$ \\
\hline
\end{tabular}

Table G.3: Individual experimental parameters for 6 arborized neurons in culture.

\begin{tabular}{|c|c|c|c|c|c|c|c|}
\hline & $\begin{array}{c}R_{m} \\
{[M \Omega]}\end{array}$ & $\begin{array}{c}\tau_{m} \\
{[m s]}\end{array}$ & $\begin{array}{c}A_{w} \\
{[M \Omega]}\end{array}$ & $\begin{array}{c}\nu_{w T} \\
{[m s]}\end{array}$ & $\begin{array}{c}R_{\text {asymp }}[M \Omega] \\
{[M \Omega}\end{array}$ & $\begin{array}{c}\sigma_{d}^{\exp } \\
{[M \Omega]}\end{array}$ & $\begin{array}{c}\sigma_{r}^{\text {exp }} \\
{[M \Omega]}\end{array}$ \\
\hline $\mathbf{A}$ & 3150 & 30 & 1050 & 0.02 & 2 & 2.0 & 223.0 \\
\hline B & 2100 & 30 & 1260 & 0.01 & 0 & 2.5 & 129.0 \\
\hline $\mathbf{C}$ & 1200 & 25 & 1000 & 0.03 & 0 & 2.0 & 97.0 \\
\hline $\mathbf{D}$ & 1200 & 35 & 1200 & 0.02 & 3 & 3.8 & 101.0 \\
\hline $\mathbf{E}$ & 1200 & 35 & 1200 & 0.03 & 3 & 3.1 & 108.0 \\
\hline $\mathbf{F}$ & 1500 & 35 & 1500 & 0.02 & 0 & 4.1 & 131.0 \\
\hline
\end{tabular}

Table G.4: Individual parameters obtained from fits for 6 arborized neurons in culture from Table G.3. $\sigma_{r}^{e x p}$ and $\sigma_{d}^{e x p}$ are respectively the mean square error of resistive and diffusive models with respect to the experimental data. 

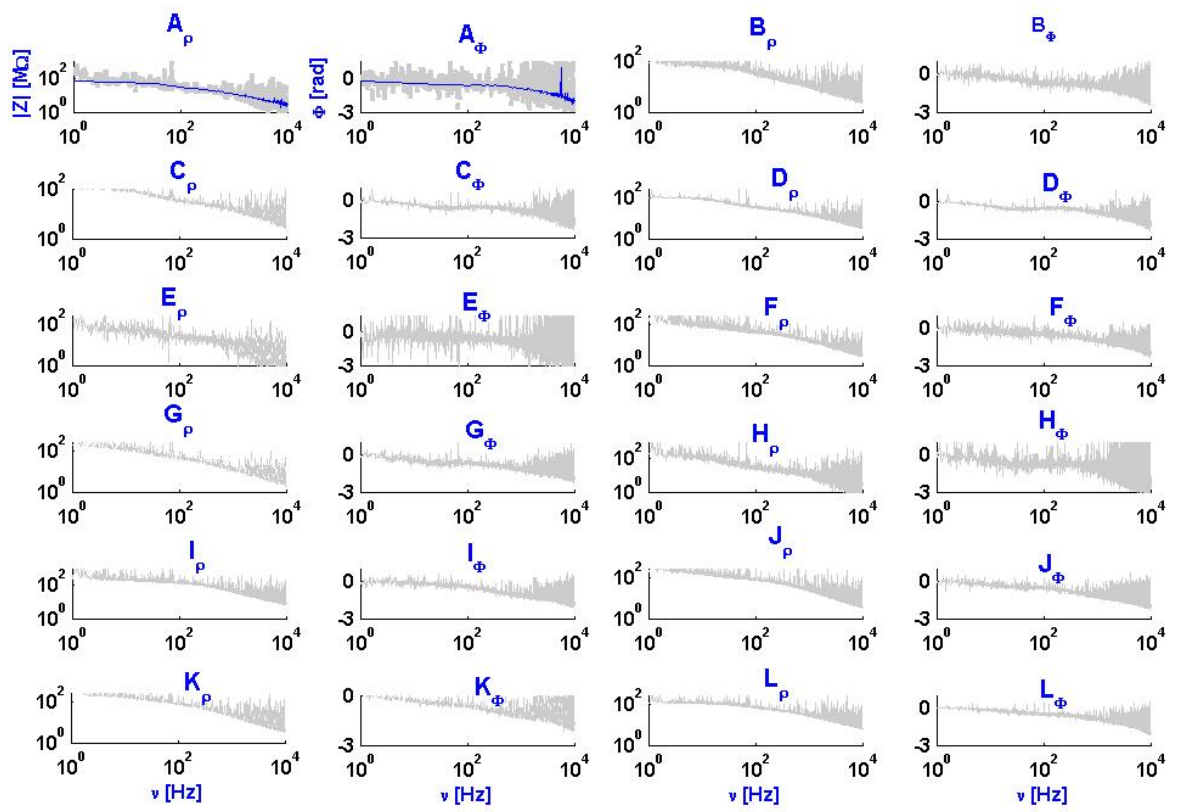

Figure G.5: Experimental measurements between intracellular and extracellular electrodes for 9 arborized neurons in brain slices, as shown in Tables G.5 and G.6. The blue curves are the cubic spline fits of the experimental data.
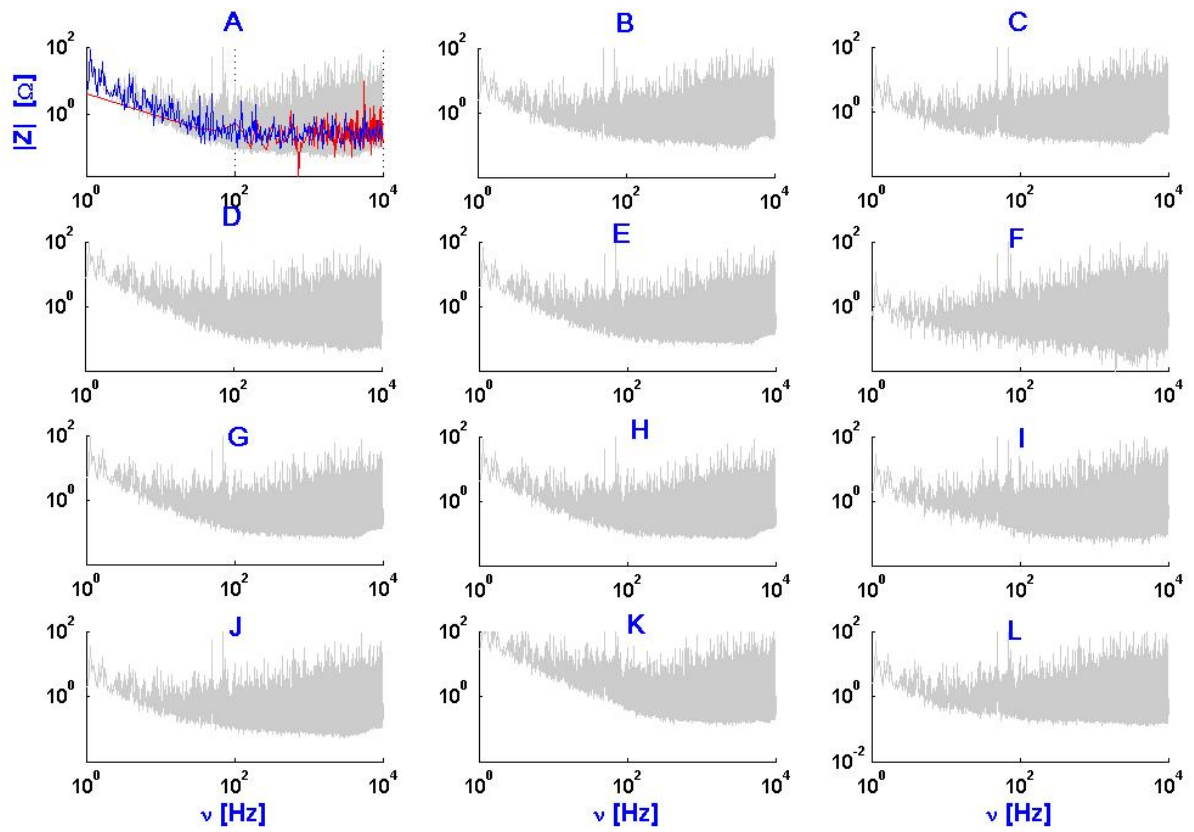

Figure G.6: Experimental measurements between extracellular and ground for arborized neurons in slices, as shown in Tables G.5 and G.6. The blue curves are cubic spline fits to the logarithm of the experimental data, and the red curves are the direct cubic spline fits of the data. 


\begin{tabular}{|c|c|c|c|c|}
\hline & $\begin{array}{c}V_{m}(\text { mean } \pm \\
\text { standard deviation }) \\
\text { during white } \\
\text { noise injection }(\mathrm{mV})\end{array}$ & $\begin{array}{l}\sigma \text { of white } \\
\text { noise } \\
\text { current } \\
(\mathrm{pA})\end{array}$ & $\begin{array}{l}\text { Number } \\
\text { of sweeps }\end{array}$ & $\begin{array}{l}\text { Membrane } \\
\text { resistance }(\mathrm{M} \Omega) \\
\text { Membrane time } \\
\text { constant }(\mathrm{ms})\end{array}$ \\
\hline A : $2018121204(\mathrm{MSN})$ & $-75.2 \pm 1.1$ & 20 & 1 & $\begin{array}{l}67 \\
6.0\end{array}$ \\
\hline B : $2018121205(\mathrm{FS})$ & $-74.8 \pm 1.2$ & 20 & 10 & $\begin{array}{l}53 \\
5.5\end{array}$ \\
\hline C : $2018122701(\mathrm{FS})$ & $-78.2 \pm 1.3$ & 20 & 15 & $\begin{array}{l}124 \\
5.6\end{array}$ \\
\hline D : $2019021202(\mathrm{MSN})$ & $-75.8 \pm 1.3$ & 20 & 6 & $\begin{array}{l}83 \\
4.2\end{array}$ \\
\hline E : 2019021203 (MSN) & $-73.9 \pm 1.7$ & 20 & 59 & $\begin{array}{l}85 \\
4.0\end{array}$ \\
\hline $\mathbf{F}: 2019021302^{*}(\mathrm{MSN})$ & $-69.1 \pm 1.8$ & 20 & 13 & $\begin{array}{l}64 \\
7.7\end{array}$ \\
\hline G : $2019021304^{*}(\mathrm{MSN})$ & $-69.3 \pm 1.7$ & 20 & 10 & $\begin{array}{l}128 \\
9.5\end{array}$ \\
\hline H : 2019021401 (MSN) & $-64.6 \pm 1.4$ & 10 & 5 & $\begin{array}{l}124 \\
8.4\end{array}$ \\
\hline I : $2019021801(\mathrm{MSN})$ & $-80.1 \pm 2.7$ & 10 & 31 & $\begin{array}{c}92 \\
2.8\end{array}$ \\
\hline $\begin{array}{c}\text { J : } 2019021802 \\
\text { (Cholinergic interneuron) }\end{array}$ & $-73.3 \pm 1.6$ & 10 & 35 & $\begin{array}{l}158 \\
6.3\end{array}$ \\
\hline $\mathbf{K}: 2019021803^{*}(\mathrm{MSN})$ & $-67.1 \pm 1.6$ & 10 & 8 & $\begin{array}{l}206 \\
7.9\end{array}$ \\
\hline L : 2019021901 (MSN) & $-76.8 \pm 1.4$ & 10 & 49 & $\begin{array}{c}44 \\
3.4\end{array}$ \\
\hline
\end{tabular}

Table G.5: Individual experimental parameters for 9 arborized neurons in brain slices (MSN: medium-sized spiny neuron; FS: fast-spiking interneuron). 


\begin{tabular}{|c|c|c|c|c|c|c|c|c|c|c|}
\hline & $\bar{R}_{m}$ & $R_{m}$ & $\tau_{m}$ & $A_{w}^{a}$ & $\nu_{w T}^{a}$ & $A_{w}^{b}$ & $\nu_{w T}^{b}$ & $R_{\text {asymp }}$ & $\sigma_{d}^{\text {exp }}$ & $\sigma_{r}^{\text {exp }}$ \\
& {$[M \Omega]$} & {$[M \Omega]$} & {$[m s]$} & {$[M \Omega]$} & {$[H z]$} & {$[M \Omega]$} & {$[\mathrm{Hz}]$} & {$[M \Omega]$} & {$[M \Omega]$} & {$[M \Omega]$} \\
\hline $\mathbf{A}$ & 120 & 48 & 5 & 48 & 5 & 24 & 40 & 1 & 2.1 & 6.0 \\
\hline $\mathbf{B}$ & 120 & 50 & 5 & 50 & 5 & 24 & 40 & 4 & 2.3 & 6.6 \\
\hline $\mathbf{C}$ & 140 & 47 & 5 & 47 & 5 & 40 & 60 & 4 & 2.3 & 5.2 \\
\hline $\mathbf{D}$ & 140 & 48 & 5 & 48 & 1 & 40 & 60 & 2 & 2.4 & 5.1 \\
\hline $\mathbf{E}$ & 140 & 48 & 2.5 & 48 & 1 & 40 & 60 & 1 & 2.0 & 6.0 \\
\hline $\mathbf{F}$ & 140 & 48 & 5 & 48 & 1 & 40 & 60 & 4 & 5.5 & 11.0 \\
\hline $\mathbf{G}$ & 256 & 90 & 20 & 90 & 1 & 75 & 40 & 1 & 6.3. & 9.5 \\
\hline $\mathbf{H}$ & 256 & 90 & 20 & 90 & 0.1 & 75 & 10 & 1 & 3.0 & 10.1 \\
\hline $\mathbf{I}$ & 426 & 150 & 1 & 150 & 1 & 125 & 20 & 1 & 28.1 & 72.0 \\
\hline $\mathbf{J}$ & 320.5 & 90 & 10 & 90 & 20 & 125 & 60 & .5 & 6.1 & 26.3 \\
\hline $\mathbf{K}$ & 320.5 & 90 & 10 & 90 & 20 & 75 & 60 & .5 & 6.1 & 26.3 \\
\hline $\mathbf{L}$ & 320.5 & 48 & 1 & 48 & 45 & 40 & 80 & 3 & 6.3 & 15.3 \\
\hline
\end{tabular}

Table G.6: Individual parameters obtained from the fits for arborized neurons in brain slices from Table G.5. The total resistance for $\nu=0$ is equal to $\bar{R}_{m}=$ $R_{m}+A_{w}^{a}+A_{w}^{b}+R_{\text {asymp }} \cdot \sigma_{r}^{\text {exp }}$ and $\sigma_{d}^{\text {exp }}$ are the mean square errors of the resistive and diffusive models relative to experimental data, respectively.

The extracellular-to-ground measurements are similar in all preparations and exhibit a similar frequency dependence. All fits show that the impedance modulus of the extracellular medium is of the order of that of ACSF.

However, this is not the case for the intracellular-to-extracellular measurements in the different preparations. For all cells and for all preparations, the diffusive model fits better the experimental data.

Finally, for the different preparations, the experimental measurements (membrane resistance and membrane time constant) shown in Table G.1 are different from that displayed in Table G.2. This difference shows that the evaluation of the membrane time constant using a current pulse leads to different membrane time constants as those evaluated from direct fitting of the impedance. This aspect is examined in more detail in the following (Appendix H). 


\section{H Voltage between two points for a square current pulse}

In this appendix, we calculate the voltage between two points when the injected current is a square pulse.

We model a pulse of current as follows:

$$
I^{g}=h[H(t)-H(t-\Delta)]
$$

where $\Delta>0$ is duration of the current pulse, and $H$ is the Heaviside function.

The most general linear relation between current and voltage is given by the following expression:

$$
V(t)=\int_{-\infty}^{+\infty} Z\left(t-t^{\prime}\right) I^{g}\left(t^{\prime}\right) d t^{\prime}=\int_{-\infty}^{+\infty} Z\left(t^{\prime}\right) I^{g}\left(t-t^{\prime}\right) d t^{\prime}
$$

It follows that the derivative of the voltage is given by:

$$
\frac{d V}{d t}=h\left[\int_{0^{-}}^{+\infty} Z\left(t^{\prime}\right) \delta\left(t-t^{\prime}\right) d t^{\prime}-\int_{\Delta^{-}}^{+\infty} Z\left(t^{\prime}\right) \delta\left(t-\Delta-t^{\prime}\right) d t^{\prime}\right] .
$$

with $\frac{d I}{d t}=h[\delta(t)-\delta(t-\Delta)]$.

Thus, we obtain the following inequality:

$$
\frac{d V}{d t}=h[Z(t)-Z(t-\Delta)]
$$

According to the complex Fourier transform,

$$
\left\{\begin{array}{l}
Z(t)=P P\left[\frac{1}{2 \pi} \int_{-\infty}^{+\infty} Z(\omega) e^{i \omega t} d \omega\right] \quad(a) \\
V(t)=P P\left[\frac{1}{2 \pi} \int_{-\infty}^{+\infty} V(\omega) e^{i \omega t} d \omega\right]
\end{array}\right.
$$

where PP means the principal part of the integral.

In a diffusive medium, we have:

$$
Z(\omega)=\frac{R_{m}}{1+i \omega \tau_{m}}+\frac{A_{w}}{1+\sqrt{i \omega \tau_{w T}}}+R_{a s y m p}
$$

where $\tau_{w T}=1 / \omega_{w T}$. We calculate $\frac{d V}{d t}$ from Equations H.4 and H.5.

Applying Eq. H.5(a) on the right-hand part of Expression H.6 gives [36]).

$$
\frac{d V}{d t}=h H(t) F(t)-h H(t-\Delta) F(t-\Delta)
$$


where

$$
F(t)=\frac{R_{m}}{\tau_{m}} e^{-\frac{t}{\tau_{m}}}+\frac{A_{w}}{\tau_{w T}}\left[\frac{1}{\sqrt{\pi} \sqrt{\frac{t}{\tau_{w T}}}}-e^{\frac{t}{\tau_{w T}}} \operatorname{erfc}\left(\sqrt{\frac{t}{\tau_{w T}}}\right)\right]+R_{\text {asymp }} \delta(t)
$$

The function $e^{x} \cdot \operatorname{erf} c(\sqrt{x})$ for $x \geq 0$ is a real and positive monotonously decreasing function which tends to zero at infinite. At $x=0$, it is equal to 1 .

Because the experimental measurements shown here indicates that $\tau_{m}<<\tau_{w T}$, we develop $\mathrm{F}(\mathrm{t})$ in series around zero to evaluate this function when $t<\tau_{w T}$. We obtain

$$
\left\{\begin{array}{ccc}
e^{t / \tau_{w T}} & = & 1+\frac{t}{\tau_{w T}}+0(2) \\
\operatorname{erfc}\left(\sqrt{t / \tau_{w T}}\right) & = & 1-\frac{1}{\sqrt{\pi}}\left[2\left(\frac{t}{\tau_{w T}}\right)^{1 / 2}-\frac{2}{3}\left(\frac{t}{\tau_{w T}}\right)^{3 / 2}+0(5 / 2)\right]
\end{array}\right.
$$

We then write the following equalities:

$F(t)=\frac{R_{m}}{\tau_{m}} e^{-\frac{t}{\tau_{m}}}-\frac{A_{w}}{\tau_{w T}}\left[1+\frac{t}{\tau_{w T}}\right]+\frac{A_{w}}{\tau_{w T} \sqrt{\pi}}\left[\frac{t^{-1 / 2}}{\tau_{w T}^{-1 / 2}}+2 \frac{t^{+1 / 2}}{\tau_{w T}^{+1 / 2}}\right]+R_{a s y m p} \delta(t)+0(3 / 2)$

and

$$
G(t)=\int_{0^{-}}^{t} F\left(t^{\prime}\right) d t^{\prime}=R_{m}\left(1-e^{-\frac{t}{\tau_{m}}}\right)-A_{w} \frac{t}{\tau_{w T}}+\frac{2 A_{w}}{\sqrt{\pi}} \frac{t^{1 / 2}}{\tau_{w T}^{1 / 2}}+R_{a s y m p}+0(3 / 2)
$$

The voltage for $V\left(0^{-}\right)=0$ at $t=0$ and $t \geq \Delta$ is given by (see H.7)

$$
V(t)=\overbrace{h \int_{0}^{\Delta} F\left(t^{\prime}\right) d t^{\prime}}^{A}+\overbrace{h \int_{\Delta}^{t} F\left(t^{\prime}\right) d t^{\prime}}^{B}-\overbrace{h \int_{\Delta}^{t} F\left(t^{\prime}-\Delta\right) d t^{\prime}}^{C}
$$

which gives

$$
\left\{\begin{array}{lll}
A(\Delta ; 0) & = & h[G(\Delta)-G(0)] \\
B(t ; \Delta) & = & h[G(t)-G(\Delta)] \\
C(t ; \Delta) & =h[G(t-\Delta)-G(0)]
\end{array}\right.
$$


Thus, we have

$$
\left\{\begin{array}{l}
A(\Delta ; 0)=h R_{m}\left[1-e^{-\frac{\Delta}{\tau_{m}}}\right]+h A_{w} \underbrace{\left[\frac{2}{\sqrt{\pi}} \frac{\Delta^{1 / 2}}{\tau_{w T}^{1 / 2}}-\frac{\Delta}{\tau_{w T}}\right]}_{>0} \\
B(t ; \Delta)=h R_{m} e^{-\frac{\Delta}{\tau_{m}}}\left[1-e^{-\frac{t-\Delta}{\tau_{m}}}\right]+h A_{w} \underbrace{\left[\frac{2}{\sqrt{\pi}} \frac{t^{1 / 2}-\Delta^{1 / 2}}{\tau_{w T}^{1 / 2}}-\frac{t-\Delta}{\tau_{w T}}\right]}_{>0} \\
C(t ; \Delta)=h R_{m}\left[1-e^{-\frac{t-\Delta}{\tau_{m}}}\right]+h A_{w} \underbrace{\left[\frac{2}{\sqrt{\pi}} \frac{(t-\Delta)^{1 / 2}}{\tau_{w T}^{1 / 2}}-\frac{t-\Delta}{\tau_{w T}}\right]}_{>0}
\end{array}\right.
$$

$A(\Delta)$ corresponds to the voltage reached at time $\Delta$ ( $\Delta$ is the duration of the current pulse). $A(\Delta)$ depends on $A_{w}$. If $A_{w}=0$, the voltage is equal to that of a resistive model. In the case of a diffusive model $A_{w} \neq 0$ is positive because $\frac{\Delta}{\tau_{w T}}<\frac{t}{\tau_{w T}}<1$. Consequently, the voltage as a function of time is given by:

$$
V(t)=\overbrace{h R_{m}\left[1-e^{-\frac{\Delta}{\tau_{m}}}\right] e^{-\frac{t-\Delta}{\tau_{m}}}}^{\text {resistive model }}+h A_{w}\left[-\frac{\Delta}{\tau_{w T}}+\frac{2}{\sqrt{\pi}}\left(\frac{t^{1 / 2}}{\tau_{w T}^{1 / 2}}-\frac{(t-\Delta)^{1 / 2}}{\tau_{w T}^{1 / 2}}\right)\right]+0(3 / 2)
$$

when $t>\Delta$ and $t<\tau_{w T}$.

Finally, the last expression is equivalent to the second-order approximation in $\sqrt{t}$ of the expression

$V(t)=\overbrace{h R_{m}\left[1-e^{-\frac{\Delta}{\tau_{m}}}\right] e^{-\frac{t-\Delta}{\tau_{m}}}}^{\text {resistive model }}-h A w\left[1-e^{-\frac{\Delta}{\tau_{w T}}}\right] e^{-\frac{t-\Delta}{\tau_{w T}}}+\frac{2}{\sqrt{\pi}} h A_{w}\left[1-e^{\left(\sqrt{\frac{t-\Delta}{\tau_{w T}}}-\sqrt{\frac{t}{\tau_{w T}}}\right)}\right] e^{-\sqrt{\frac{t-\Delta}{\tau_{w T}}}+0(3 / 2)}$

when $t>\Delta$ and $t<\tau_{w T}$. We can also develop the third term of expression H.16 as a series of exponentials linear in $t$ (using Newton binomial) with a combination of exponentials with different relaxation times. 


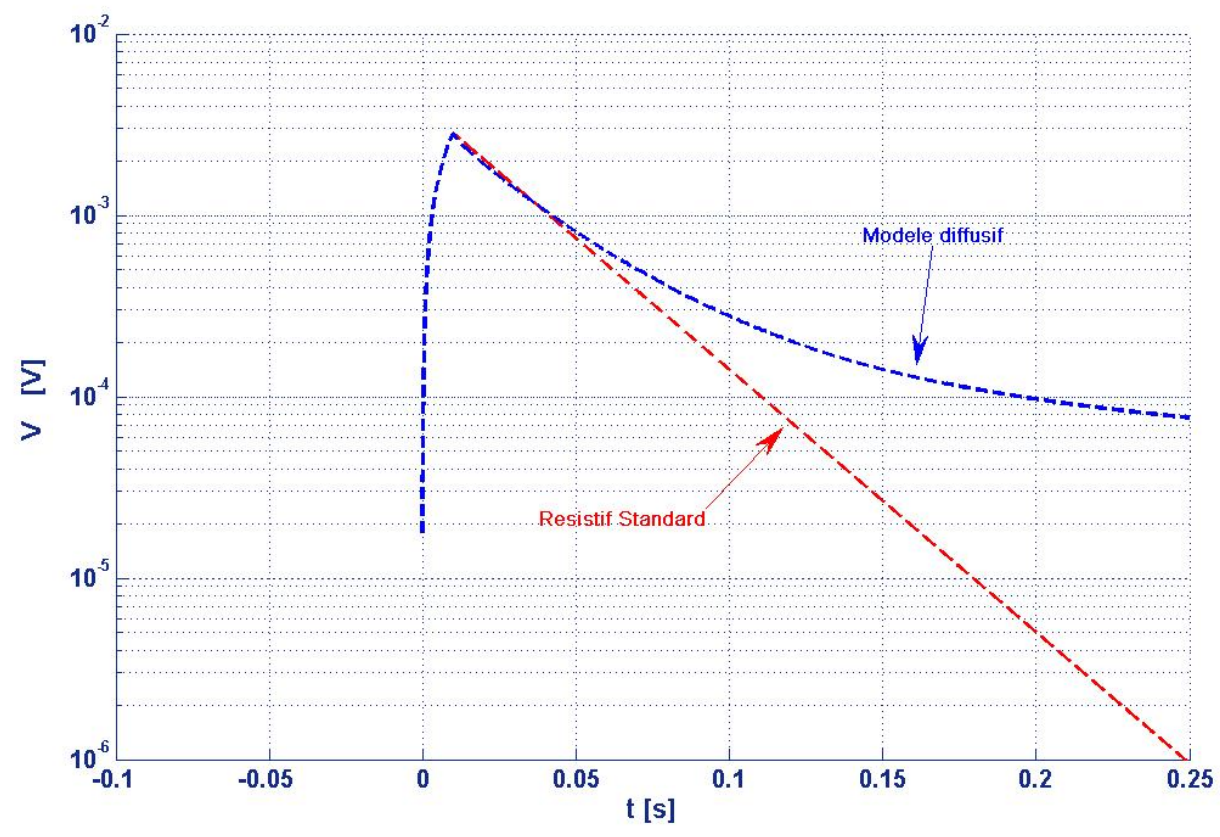

Figure H.1: Voltage relative to rest as a function of time during the injection of a current pulse of $10 p A$ and $10 \mathrm{~ms}$ duration. The membrane time constant was $\tau_{m}=30 \mathrm{~ms}$ and the impedance of the extracellular medium + membrane is of $1000 M \Omega$ at null frequency. The red curve shows the prediction of the resistive model, while the blue curve shows the resistive model (with a threshold frequency of $\nu_{w T}=0.5 \mathrm{~Hz}$ and $\left.A_{w}=R_{m}\right)$.

Consequently, the method of current pulse injection and the linearization method give a different slope in a semi-log graph, according to the type of model. For a resistive model, the slope is $-1 / \tau_{m}$, and this allows to directly estimate the membrane time constant, as classically performed. However, for a diffusive model, the slope is slightly variable and smaller than the resistive model. It depends on $\tau_{m}, \nu_{w T}$ and on the ratio $R_{m} / A_{w}$. For $t>\Delta$, one part of the voltage, $V_{a}\left(V=V_{a}+V_{b}\right)$, attenuates according to the resistive model, while the other part, $V_{b}$, attenuates slower and depends on $\nu_{w T}$. This explains why the membrane time constant seems larger with the pulse or linearization method, compared to the fitting of experimental measurements (see Appendix G). The divergence originates from the pulse or linearization methods according to a resistive model. 